\title{
Nucleon matrix elements using the variational method in lattice QCD
}

\author{
J. Dragos, ${ }^{1}$ R. Horsley, ${ }^{2}$ W. Kamleh, ${ }^{1}$ D. B. Leinweber, ${ }^{1}$ Y. Nakamura, ${ }^{3}$ \\ P. E. L. Rakow, ${ }^{4}$ G. Schierholz, ${ }^{5}$ R. D. Young, ${ }^{1,6}$ and J. M. Zanotti ${ }^{1}$ \\ ${ }^{1}$ CSSM, Department of Physics, The University of Adelaide, Adelaide, South Australia 5005, Australia \\ ${ }^{2}$ School of Physics and Astronomy, University of Edinburgh, Edinburgh EH9 3JZ, United Kingdom \\ ${ }^{3}$ RIKEN Advanced Institute for Computational Science, Kobe, Hyogo 650-0047, Japan \\ ${ }^{4}$ Theoretical Physics Division, Department of Mathematical Sciences, \\ University of Liverpool, Liverpool L69 3BX, United Kingdom \\ ${ }^{5}$ Deutsches Elektronen-Synchrotron DESY, 22603 Hamburg, Germany \\ ${ }^{6}$ CoEPP, Department of Physics, University of Adelaide, Adelaide, South Australia 5005, Australia
}

(Received 17 June 2016; published 25 October 2016)

\begin{abstract}
The extraction of hadron matrix elements in lattice QCD using the standard two- and three-point correlator functions demands careful attention to systematic uncertainties. One of the most commonly studied sources of systematic error is contamination from excited states. We apply the variational method to calculate the axial vector current $g_{A}$, the scalar current $g_{S}$, the scalar current $g_{T}$ and the quark momentum fraction $\langle x\rangle$ of the nucleon and we compare the results to the more commonly used summation and twoexponential fit methods. The results demonstrate that the variational approach offers a more efficient and robust method for the determination of nucleon matrix elements.
\end{abstract}

DOI: 10.1103/PhysRevD.94.074505

\section{INTRODUCTION}

Modern lattice QCD simulations are making significant advances towards the direct comparison with experimental results for a range of hadronic observables. Therefore there is an increasing demand for numerical studies to quantify all uncertainties, both statistical and systematic. In this present work, we focus specifically on the systematic uncertainty associated with excited-state contamination in baryon matrix elements. The presence of the weak signal-to-noise behavior makes the study of baryon three-point functions particularly sensitive to excited-state contamination. In practice, there is a persistent trade-off to keep the source-sink time separation short enough to provide a statistically significant signal, while desiring a long enough separation to suppress excited states.

In this study we investigate a range of techniques for addressing excited-state contamination in baryon matrix elements. Our focus is on the variational method, which has seen tremendous success in spectroscopy studies [1-7], in addition to some applications in hadronic matrix elements [8-15]. We then compare the variational method to the popular "two-exponential fit" and "summation" methods seen in the literature [13-21]. The observables we choose to study are the nucleon axial vector charge $g_{A}$, the nucleon scalar charge $g_{S}$, the nucleon tensor charge $g_{T}$ and the quark momentum fraction $\langle x\rangle$ for the nucleon. The latter two have previously been identified as being particularly sensitive to excited-state contamination. The results of our analysis demonstrate, for all three quantities considered, that the variational method offers improved reliability in comparison to the summation and twoexponential fit methods.
The structure of this paper is as follows. Section II contains an explanation of the gauge field configurations used along with our method for creating correlation functions. Section III outlines the application of the variational approach to three-point functions, including a prescription for optimizing the sequential source through the sink inversion. Section IV summarizes the implementation of the summation method and two-exponential fit. Section V presents the numerical results from this paper. Section VI summarizes our findings and discusses the contrasting features of the various techniques presented. Section VII provides concluding remarks and the future outlook.

\section{LATTICE DETAILS}

\section{A. Simulation details}

Simulations were performed on a $32^{3} \times 64$-dimensional ensemble with a pion mass of $460 \mathrm{MeV}$ and a lattice spacing of $0.074 \mathrm{fm}$ [22-24]. This ensemble corresponds to the SU(3)-symmetric point, where $m_{u}=m_{d}=m_{s}$ with $\kappa=0.120900$, which has been tuned to be close to the physical, average light-quark mass $\bar{m}=\frac{1}{3}\left(m_{u}+m_{d}+m_{s}\right)$ [24]. The simulation uses a clover action comprising a stout smeared fermion action along with the tree-level Symanzik improved gluon action. We perform $\mathcal{O}(1000)$ measurements on $\mathcal{O}(1800)$ trajectories, with multiple source location to remove autocorrelations. The renormalization constants $Z_{A}=0.8728(6)(27), Z_{S}^{\overline{\mathrm{MS}}}=0.682(6)(18)$ and $Z_{T}^{\overline{\mathrm{MS}}}=0.9945(010)(035)$ at $2 \mathrm{GeV}$ have been reported in Ref. [25], whereas $\langle x\rangle$ remains unrenormalized in the present work. 
A fixed boundary condition in the Euclidean time dimension and periodic boundary conditions in the spatial dimensions are chosen for this calculation. As outlined in the next section, we employ the sequential source through the sink method to compute three-point functions (see [26]). Hence we are required to fixed the sink momentum $\vec{p}^{\prime}$ for which we set $\vec{p}^{\prime}=\overrightarrow{0}$. The space of all Hermitian matrices combined with zero and one derivative operators has been calculated as they require minimal computational time after the sequential propagators have been created. Although different transfer momenta have been calculated with the zero sink momentum, this paper only analyzes forward matrix elements (zero momentum transfer) and the three particular operators and spin projectors corresponding to $g_{A}, g_{T},\langle x\rangle$ and $g_{S}$ as described in Sec. V.

The smearings undertaken in later sections are gaugeinvariant Gaussian smearings which have the functional form [27]

$$
\begin{aligned}
H\left(\vec{x}^{\prime}, \vec{x}\right)= & (1-\alpha) \delta_{\vec{x} \vec{x}^{\prime}}+\cdots \frac{\alpha}{6} \sum_{\hat{i}}\left\{\delta_{\vec{x}\left(\vec{x}^{\prime}-\hat{i}\right)} \mathcal{U}_{\hat{i}}(\vec{x})\right. \\
& \left.+\delta_{\vec{x}\left(\vec{x}^{\prime}+\hat{i}\right)} \mathcal{U}_{\hat{i}}^{\dagger}(\vec{x}-\hat{i})\right\},
\end{aligned}
$$

and are applied iteratively to the source and sink quark field.

We take $\alpha=0.7$ and then by repeated application of this smearing operator $N_{\text {smear }}$ times we generate quark source and sink distributions of different spatial sizes. To form our variational basis we solved our quark propagators for 32, 64 and 128 sweeps of smearing which correspond to root mean square radii of $0.248 \mathrm{fm}, 0.351 \mathrm{fm}$ and $0.496 \mathrm{fm}$ respectively.

To get an extensive range of source-sink separation times for the study of the summation method, we have performed the sequential-source inversions at source-sink separations of 10, 13, 16, 19 and 22 time slices. In physical units, this corresponds to the range $0.74-1.63 \mathrm{fm}$. This extended range is primarily at our reference source smearing of 32 . The full ensemble of inversions performed in this study is indicated in Table I.

\section{B. Two-point and three-point correlation functions}

We follow the standard notation for a nucleon two-point correlation function with momentum $\vec{p}$ at Euclidean time $t$ :

TABLE I. Table showing all smearing and source-sink separations undertaken in this paper.

\begin{tabular}{lllllll}
\hline \hline$N_{\text {smear }}$ & $t$ & 10 & 13 & 16 & 19 & 22 \\
\hline 32 & & $\star$ & $\star$ & $\star$ & $\star$ & $\star$ \\
64 & & & $\star$ & & & \\
128 & & & $\star$ & & & \\
Variational & & & $\star$ & $\star$ & & \\
\hline \hline
\end{tabular}

$$
G_{2}(\Gamma ; \vec{p}, t)=\sum_{\vec{x}} e^{-i \vec{p} \cdot \vec{x}} \operatorname{Tr}\{\Gamma\langle\Omega|\chi(\vec{x}, t) \bar{\chi}(0)| \Omega\rangle\},
$$

where $\chi$ is a proton interpolating operator and $\Gamma=$ $\Gamma_{4} \equiv \frac{I+\gamma_{4}}{2}$ is used to project onto positive parity states. This equation reduces to the following:

$$
G_{2}(\vec{p}, t) \equiv G_{2}\left(\Gamma=\Gamma_{4} ; \vec{p}, t\right)=\sum_{\alpha} e^{-E_{\vec{p}}^{\alpha} t} \bar{Z}_{\vec{p}}^{\alpha} Z_{\vec{p}}^{\alpha},
$$

where $Z_{\vec{p}}^{\alpha}$ and $\bar{Z}_{\vec{p}}^{\alpha}$ are momentum-dependent constants of state $\alpha$ related to the coupling strengths of the operators to their energy eigenstates of energy $E_{\vec{p}}^{\alpha}$. For three-point correlators, we repeat with an inserted current operator $O(\vec{y}, \tau)$ at some intermediate time $\tau$ :

$$
\begin{aligned}
& G_{3}\left(\Gamma ; \vec{p}^{\prime}, t ; \vec{q}, \tau ; O\right) \\
& \quad=\sum_{\vec{x}, \vec{y}} e^{-i \vec{p}^{\prime} \cdot \vec{x}} e^{i \vec{q} \cdot \vec{y}} \operatorname{Tr}\{\Gamma\langle\Omega|\chi(\vec{x}, t) O(\vec{y}, \tau) \bar{\chi}(0)| \Omega\rangle\} .
\end{aligned}
$$

In this notation, $\vec{p}^{\prime}$ is the momentum of the final state, $\vec{p}$ is the momentum of the initial state and the momentum transferred to the nucleon by the operator $O$ is defined as $\vec{q} \equiv \vec{p}^{\prime}-\vec{p}$.

Reducing the three-point correlator in a similar way to the two-point correlator, Eq. (3), we have

$$
\begin{aligned}
& G_{3}\left(\Gamma ; \vec{p}^{\prime}, t ; \vec{q}, \tau ; O\right) \\
& \quad=\sum_{\alpha, \beta} e^{-E_{\vec{p}^{\prime}}^{\alpha}(t-\tau)} e^{-E_{\vec{p}}^{\beta} \tau} \bar{Z}_{\vec{p}^{\prime}}^{\alpha} Z_{\vec{p}}^{\beta} F F\left(E_{\vec{p}}^{\beta} \rightarrow E_{\vec{p}^{\prime}}^{\alpha}, \Gamma, O\right),
\end{aligned}
$$

defining the "FF" function as

$$
\begin{aligned}
& \operatorname{FF}\left(E_{\vec{p}}^{\beta} \rightarrow E_{\vec{p}^{\prime}}^{\alpha}, \Gamma, O\right) \\
& \quad \equiv \operatorname{Tr}\left\{\Gamma\left(\frac{p^{\prime}+m^{\alpha}}{2 E_{\vec{p}^{\prime}}^{\alpha}}\right) \mathcal{J}_{O}\left(q^{2}\right)\left(\frac{\not p+m^{\beta}}{2 E_{\vec{p}}^{\beta}}\right)\right\},
\end{aligned}
$$

where $E_{\vec{p}^{\prime}}^{\alpha}$ and $E_{\vec{p}}^{\beta}$ are the source and sink energies, respectively, referring to the state indices $\alpha$ and $\beta$, with momenta $\vec{p}^{\prime}$ and $\vec{p}$. $\mathcal{J}_{O}\left(q^{2}\right)$ is the appropriate form factor combination for the particular operator $\mathcal{O}$ chosen. For example, choosing $\mathcal{O}=i \gamma_{3} \gamma_{5}$ corresponds to

$$
\mathcal{J}_{i \gamma_{3} \gamma_{5}}\left(q^{2}\right)=i \gamma_{5} \gamma_{3} G_{A}\left(q^{2}\right)-\frac{\gamma_{5} q_{3}}{2 m} G_{P}\left(q^{2}\right),
$$

where $G_{A}$ and $G_{P}$ are the axial and induced pseudoscalar form factors, respectively.

Once $G_{2}$ and $G_{3}$ are obtained, we can define the combination to remove the exponential time dependence and wave function overlap factors: 


$$
\begin{aligned}
& R\left(\Gamma ; \vec{p}^{\prime}, t ; \vec{q}, \tau ; O\right) \\
& \quad \equiv \frac{G_{3}\left(\Gamma ; \vec{p}^{\prime}, t ; \vec{q}, \tau ; O\right)}{G_{2}\left(\vec{p}^{\prime}, t\right)} \sqrt{\frac{G_{2}\left(\vec{p}^{\prime}, \tau\right) G_{2}\left(\vec{p}^{\prime}, t\right) G_{2}(\vec{p}, t-\tau)}{G_{2}(\vec{p}, \tau) G_{2}(\vec{p}, t) G_{2}\left(\vec{p}^{\prime}, t-\tau\right)}} .
\end{aligned}
$$

Due to the exponential time dependence in the two- and three-point correlators, ground-state dominance will occur at large times $\tau \gg 0$ and $t \gg \tau$. Hence, the FF function can be extracted by taking large $\tau$ and $t$ limits:

$$
\begin{aligned}
R\left(\Gamma ; \vec{p}^{\prime}, t ; \vec{q}, \tau ; O\right) \stackrel{\tau \gg 0, t \gg \tau}{\longrightarrow} A\left(\vec{p}, \vec{p}^{\prime}, m^{\alpha}, m^{\beta}\right) \\
\times \operatorname{FF}\left(E_{\vec{p}}^{\beta} \rightarrow E_{\vec{p}^{\prime}}^{\alpha}, \Gamma, O\right),
\end{aligned}
$$

where $A\left(\vec{p}, \vec{p}^{\prime}, m^{\alpha}, m^{\beta}\right)$ is a known kinematical constant.

\section{VARIATIONAL METHOD}

In the previous section we showed how to determine the ground-state properties by studying the large time behavior of two- and three-point correlation functions. As is well known, the signal-to-noise ratio of nucleon correlation functions decreases significantly at large times. Hence with finite statistics, it is often necessary to find a balance between large source-current-sink time separations and quality of signal. To help alleviate this problem, it would be advantageous if one were able to reduce the contributions from excited states at early times in order to facilitate the extraction of ground-state properties at early times. The variational method has proven to be a robust and useful tool for studying two-point correlators in this respect [1-7]. Recently, this approach has been extended to three-point correlators, specifically aiming to reduce the effect of excited-state contamination in hadronic matrix elements [8-15].

Once a basis of states is obtained that contains different couplings to different energy levels, a variational analysis can be undertaken to produce correlation functions that couple strongly to the ground state. Given the significant signal/noise problem for baryon correlators, any reduction in the time required to saturate the ground state can give significant advantage in the study of three-point correlators.

We present our notation for the variational approach, following a format similar to that described in Ref. [11]. Ideally, the improved two-point correlation function isolating the generic state $\beta$ is given by

$G_{2}^{\beta}(\Gamma ; \vec{p}, t)=\sum_{\vec{x}} e^{-i \vec{p} \cdot \vec{x}} \operatorname{Tr}\left\{\Gamma\left\langle\Omega\left|\phi^{\beta}(x, \vec{p}) \bar{\phi}^{\beta}(0, \vec{p})\right| \Omega\right\rangle\right\}$,

where $\phi^{\beta}(x, \vec{p})$ and $\bar{\phi}^{\beta}(0, \vec{p})$ are constructed as a linear combination of our basis of operators:

$$
\begin{aligned}
\phi^{\beta}(x, \vec{p}) & =\sum_{i} v_{i}^{\beta}(\vec{p}) \chi_{i}(x), \\
\bar{\phi}^{\beta}(0, \vec{p}) & =\sum_{i} u_{i}^{\beta}(\vec{p}) \bar{\chi}_{i}(0) .
\end{aligned}
$$

If we express the correlators $G_{2}$ created over a basis $i j$ as a matrix of correlators, we can rewrite Eq. (10) as

$$
G_{2}^{\beta}(\Gamma ; \vec{p}, t)=v_{i}^{\beta}(\vec{p})\left(G_{2}\right)_{i j}(\Gamma ; \vec{p}, t) u_{j}^{\beta}(\vec{p}),
$$

which constructs a new two-point correlator that has a stronger coupling to state $\beta$. By selecting two sink times $t=t_{0}$ and $t=t_{0}+\Delta t, u$ and $v$ can be found via the solution to the following eigenvalue equations:

$v_{i}^{\beta}(\vec{p})\left[G_{2}\left(\Gamma ; \vec{p}, t_{0}+\Delta t\right) G_{2}\left(\Gamma ; \vec{p}, t_{0}\right)^{-1}\right]_{i j}=v_{j}^{\beta}(\vec{p}) \lambda^{\beta}$,

$$
\left[G_{2}\left(\Gamma ; \vec{p}, t_{0}\right)^{-1} G_{2}\left(\Gamma ; \vec{p}, t_{0}+\Delta t\right)\right]_{i j} u_{j}^{\beta}(\vec{p})=u_{i}^{\beta}(\vec{p}) \lambda^{\beta} .
$$

For the ground state $(\beta=0)$, this creates a two-point function that has an accelerated approach to the ground state over Euclidean time. For this analysis, $\left(G_{2}\right)_{i j}$ is a $3 \times 3$ matrix corresponding to 32,64 , and 128 sweeps of smearing at the source (index $i$ ) and the sink (index $j$ ). The same $u$ and $v$ found for the two-point correlators at a particular momentum can be used to estimate the threepoint correlator for state $\beta$,

$$
\begin{aligned}
& G_{3}^{\beta}\left(\Gamma ; \vec{p}^{\prime}, t ; \vec{q}, \tau ; O\right) \\
& =\sum_{\vec{x}, \vec{y}} e^{-i \vec{p}^{\prime} \cdot \vec{x}} e^{i \vec{q} \cdot \vec{y}} \operatorname{Tr}\left\{\Gamma\left\langle\Omega\left|\phi^{\beta}\left(x, \vec{p}^{\prime}\right) \mathcal{J}_{O}(\vec{y}, \tau) \bar{\phi}^{\beta}(0, \vec{p})\right| \Omega\right\rangle\right\},
\end{aligned}
$$

or rewritten over $i j$ as

$G_{3}^{\beta}\left(\Gamma ; \vec{p}^{\prime}, t ; \vec{q}, \tau ; O\right)=v_{i}^{\beta}\left(\vec{p}^{\prime}\right)\left(G_{3}\right)_{i j}\left(\Gamma ; \vec{p}^{\prime}, t ; \vec{q}, \tau ; O\right) u_{j}^{\beta}(\vec{p})$.

And lastly, we construct the same ratio as previously described in Eq. (9) which will have the FF function dependence:

$$
\begin{aligned}
& R^{\beta}\left(\Gamma ; \vec{p}^{\prime}, t ; \vec{q}, \tau ; O\right) \\
& \quad \equiv \frac{G_{3}^{\beta}\left(\Gamma ; \vec{p}^{\prime}, t ; \vec{q}, \tau ; O\right)}{G_{2}^{\beta}\left(\vec{p}^{\prime}, t\right)} \sqrt{\frac{G_{2}^{\beta}\left(\vec{p}^{\prime}, \tau\right) G_{2}^{\beta}\left(\vec{p}^{\prime}, t\right) G_{2}^{\beta}(\vec{p}, t-\tau)}{G_{2}^{\beta}(\vec{p}, \tau) G_{2}^{\alpha}(\vec{p}, t) G_{2}^{\beta}\left(\vec{p}^{\prime}, t-\tau\right)}} .
\end{aligned}
$$

For the following results, a set of $t_{0}$ and $\Delta t$ were analyzed, and $t_{0}=2$ and $\Delta t=2$ were chosen; however minimal variation was observed for other choices as seen in Fig. 4 in Sec. V B. 


\section{A. Smearing the sink}

As the variational approach we employ uses different levels of quark smearing to form our basis of operators, we first describe how to perform the standard method for smearing the sink of a three-point function before outlining our procedure for applying the variational method at the sink. Gaussian gauge-invariant smearings are applied to the source and sink of the two-point correlation function as well as the source of the three-point correlation function. To produce an equivalent smearing at the sink for the three-point correlation function, a new construction is needed as the fixed sink method does not have direct access to the operator/interpolating field at the sink.

Two-point quark propagators are defined as

$$
S_{\alpha \beta}^{a b}(x ; y)=\bar{\psi}_{\alpha}^{a}(x) \bar{\psi}_{\beta}^{b}(y),
$$

where $\bar{\psi}$ and $\psi$ are the quark creation and annihilation operators, respectively. Hence the construction for the fixed sink method is as follows. First we write the three-point function in terms of quark propagators

$$
\begin{aligned}
& G_{3}\left(\Gamma ; \vec{p}^{\prime}, t ; \vec{q}, \tau ; O\right) \\
& \quad=\sum_{\vec{y}} e^{i \vec{q} \cdot \vec{y}} \operatorname{Tr}\left\{\sigma\left(\Gamma ; \vec{p}^{\prime}, t ; y ; 0\right) \mathcal{O}(\vec{y}, \tau) S(y ; 0)\right\},
\end{aligned}
$$

where the sequential-source through the sink propagator $\sigma$, which is demonstrated in Fig. 1, is created by solving the linear equation:

$\sum_{\vec{y}} S^{-1}(x ; y) \gamma_{5} \sigma^{\dagger}\left(\Gamma ; \vec{p}^{\prime}, t ; y ; 0\right)=e^{i \vec{p}^{\prime} \cdot \vec{x}} \gamma_{5} S_{C}^{\dagger}(x ; 0)$,

with an appropriate choice of $S_{C}(x ; 0)$. The source for the inversion, $S_{C}$ (known as a "sequential source"), is the combination of all the quark propagators from the source to the sink that have no current operators attached to them.

To smear the sink properly, the term $S^{-1}(x, y)$ must be smeared at the sink as well, but we can use the same inversion calculation by not applying the smearings to this term and instead smear the source $S_{C}$ to compensate:

$$
e^{-i \vec{p}^{\prime} \cdot \vec{x}} S_{C}(x ; 0) \Rightarrow \sum_{\vec{x}^{\prime}} e^{-i \vec{p}^{\prime} \cdot \vec{x}^{\prime}} S_{C}\left(\vec{x}^{\prime}, t ; 0\right) H\left(\vec{x}^{\prime}, \vec{x}\right),
$$

where $H$ is our smearing operator used to smear the source or sink of a propagator $S$. For this paper, a gauge-invariant Gaussian smearing is undertaken as shown in Eq. (1).

\section{B. Variational method sink smearing}

Since in most cases a single $\vec{p}^{\prime}$ is chosen (usually $\vec{p}^{\prime}=\overrightarrow{0}$ ), we can reduce the computation time for the three-point correlator from $n^{2}$ to $n$ where $n$ is the number of source and sink smearings. This is done by constructing a three-point correlator as a combination of sink smearings with weights $v$ created from the variational method on the two-point correlators:

$$
\left(G_{3}\right)_{j}^{\beta}\left(\Gamma ; \vec{p}^{\prime}, t ; \vec{q}, \tau ; O\right) \equiv v_{i}^{\beta}\left(\vec{p}^{\prime}\right)\left(G_{3}\right)_{i j}\left(\Gamma ; \vec{p}^{\prime}, t ; \vec{q}, \tau ; O\right) .
$$

So when we create the fixed sink propagator $\sigma$, we can solve Eq. (21) with a smearing substitution of

$$
e^{-i \vec{p}^{\prime} \cdot \vec{x}} S_{C}\left(\vec{p}^{\prime} ; x ; 0\right) \Rightarrow \sum_{\vec{x}^{\prime}, i} e^{-i \vec{p}^{\prime} \cdot \vec{x}^{\prime}} S_{C}\left(\vec{x}^{\prime}, t ; 0\right) H_{i}\left(\vec{x}^{\prime}, \vec{x}\right) v_{i}^{\beta}\left(\vec{p}^{\prime}\right),
$$

where $H_{i}$ is the smearing operator applied an amount of times corresponding to basis index $i$ (e.g. $i=1$ might correspond to applying 32 sweeps of smearing) and $v_{i}^{\beta}\left(\vec{p}^{\prime}\right)$ is the weightings obtained from the variational method applied to the two-point correlators.

An important point to note here is that a single combination of $t_{0}$ and $\Delta t$ must be chosen from the two-point correlator as $v_{i}^{\beta}\left(\vec{p}^{\prime}\right)$ is now used in the matrix inversion calculation to create the fixed sink propagator/ correlator and is dependent on these parameters.

\section{SUMMATION AND TWO-EXPONENTIAL FIT METHODS}

Two alternative methods that have been proposed for reducing the effect of excited-state contamination in hadronic matrix element calculations are the summation and two-state fit methods.

\section{A. Summation method}

As has been used many times in the past and in recent works [16-19], a summation method can be employed in this calculation to reduce the excited-state contamination. The process proceeds by summing the ratio $R\left(\Gamma ; \vec{p}^{\prime}, t\right.$; $\vec{q}, \tau ; O)$ over operator insertion times, $\tau$ :

$$
\begin{aligned}
S\left(\Gamma ; \vec{p}^{\prime}, t ; \vec{q} ; \mathcal{O}\right)= & \sum_{\tau=\delta t}^{t-\delta t} R\left(\Gamma ; \vec{p}^{\prime}, t ; \vec{q}, \tau ; O\right) \\
\rightarrow & c+t\left\{\operatorname{FF}\left(E_{\vec{p}}^{0} \rightarrow E_{\vec{p}^{\prime}}^{0}, \Gamma, O\right)\right. \\
& \left.+\mathcal{O}\left(e^{-\min \left(\Delta E_{\vec{p}}, \Delta E_{\vec{p}^{\prime}}\right) t}\right)\right\},
\end{aligned}
$$

where $\Delta E_{\vec{p}}$ is the energy difference between the ground and first excited-state energy with momentum $\vec{p}$. The (apparent) advantage of this technique is that the correction to the matrix element is suppressed by an exponential in $t$, the full source-sink separation time. This is in contrast to the 
conventional method where the parametric suppression of excited states is given by a similar exponential of time $t-\tau$ (or $\tau$ ), which is $\sim t / 2$ in the plateau region. We allow for the slight generalization of including a $\delta t$ parameter, also considered in [19], which describes the number of current insertion results of the summation of $R$ which have been removed closest to both the source and sink. This region has the strongest statistical signal, yet provides minimal information on the ground-state matrix element. In most instances, we find the results to be largely insensitive to $\delta t$, as one might expect. But the summation method results shown later for $\langle x\rangle$ (Fig. 21) are an example where we see a statistically significant change when we vary the $\delta t$ parameter.

After performing simulations at multiple source-sink separation times, $t$, one performs a linear fit to determine $\operatorname{FF}\left(E_{\vec{p}}^{0} \rightarrow E_{\vec{p}^{\prime}}^{0}, \Gamma, O\right)$.

\section{B. Two-exponential fit method}

Multiexponential fits have also been suggested as a way of removing excited-state contamination from the determination of ground-state quantities. While proposed long ago for spectroscopy, many recent studies have attempted this in hadron matrix element calculations [13-15,18-21]. For comparative purposes, we also explore the use of a twoexponential fit. This is undertaken by expanding the twopoint and three-point functions to the second energy state and fitting to obtain the parameters of interest. Since all calculations performed as a part of this work have $\vec{p}^{\prime}=0$, the formalism can be reduced to fitting the following functions:

$$
\begin{aligned}
& G_{2}(\vec{p}, t)=\left|Z_{E_{\vec{p}}}\right|^{2} e^{-E_{\vec{p}} t}+\left|Z_{E_{\vec{p}}^{\prime}}\right|^{2} e^{-E_{\vec{p}}^{\prime} t}+\cdots, \\
& G_{3}\left(\Gamma ; \vec{p}^{\prime}, t ; \vec{q}, \tau ; O\right) \\
&=\bar{Z}_{E_{\vec{p}}} Z_{m} e^{-m t} e^{-\left(E_{\vec{p}}-m\right) \tau} \operatorname{FF}\left(E_{\vec{p}} \rightarrow m, \Gamma, \mathcal{O}\right) \\
&+\bar{Z}_{E_{\vec{p}}^{\prime}} Z_{m} e^{-m t} e^{-\left(E_{\vec{p}}^{\prime}-m\right) \tau} \operatorname{FF}\left(E_{\vec{p}}^{\prime} \rightarrow m, \Gamma, \mathcal{O}\right) \\
&+\bar{Z}_{E_{\vec{p}}} Z_{m^{\prime}} e^{-m^{\prime} t} e^{-\left(E_{\vec{p}}-m^{\prime}\right) \tau} \mathrm{FF}\left(E_{\vec{p}} \rightarrow m^{\prime}, \Gamma, \mathcal{O}\right) \\
&+\bar{Z}_{E_{\vec{p}}^{\prime}} Z_{m^{\prime}} e^{-m^{\prime} t} e^{-\left(E_{\vec{p}}^{\prime}-m^{\prime}\right) \tau} \mathrm{FF}\left(E_{\vec{p}}^{\prime} \rightarrow m^{\prime}, \Gamma, \mathcal{O}\right) \ldots,
\end{aligned}
$$

where $E_{\vec{p}}$ and $m$ now refer to the ground-state energy and mass while the primes in $E_{\vec{p}}^{\prime}$ and $m^{\prime}$ denote the first excited-state energy and mass. Taking this framework, we can fit the nucleon two-point function to the following function to determine the mass (with $\Delta E_{\vec{p}} \equiv E_{\vec{p}}^{\prime}-E_{\vec{p}}$ and $\left.\Delta m \equiv m^{\prime}-m\right)$,

$$
G_{2}(\vec{p}, t)=A_{E_{\vec{p}}} e^{-E_{\vec{p}} t}+A_{E_{\vec{p}}^{\prime}} e^{-\left(E_{\vec{p}}+\Delta E_{\vec{p}}\right) t},
$$

and we can fit the nucleon three-point function by the following function from which we are then able to extract the FF function,

$$
\begin{aligned}
& G_{3}\left(\Gamma ; \vec{p}^{\prime}, t ; \vec{q}, \tau ; O\right) \\
& =\sqrt{A_{E_{\vec{p}}} A_{m}} e^{-m t} e^{-\left(E_{\vec{p}}-m\right) \tau}\left\{B_{00}+B_{10} e^{-\Delta E_{\vec{p}} \tau}\right. \\
& \left.\quad+B_{01} e^{-\Delta m(t-\tau)}+B_{11} e^{-\Delta m t} e^{-\left(\Delta E_{\vec{p}}-\Delta m\right) \tau}\right\},
\end{aligned}
$$

where we have four free parameters in the two-point correlator for each momentum used, as well as four free parameters in the three-point correlator fit which correspond to

$$
\begin{gathered}
B_{00}=F F\left(E_{\vec{p}} \rightarrow m, \Gamma, \mathcal{O}\right), \\
B_{10}=\sqrt{\frac{A_{E_{\vec{p}}^{\prime}}}{A_{E_{\vec{p}}}} \operatorname{FF}\left(E_{\vec{p}}^{\prime} \rightarrow m, \Gamma, \mathcal{O}\right),} \\
B_{01}=\sqrt{\frac{A_{m^{\prime}}}{A_{m}}} \operatorname{FF}\left(E_{\vec{p}} \rightarrow m^{\prime}, \Gamma, \mathcal{O}\right), \\
B_{11}=\sqrt{\frac{A_{E_{\vec{p}}^{\prime}} A_{m^{\prime}}}{A_{E_{\vec{p}}} A_{m}}} \operatorname{FF}\left(E_{\vec{p}}^{\prime} \rightarrow m^{\prime}, \Gamma, \mathcal{O}\right) .
\end{gathered}
$$

For the forward matrix elements considered in this work we require only $\vec{p}=\vec{p}^{\prime}=0$, which implies $E_{\vec{p}}=m$ and $\Delta E_{\vec{p}}=\Delta m$, and hence

$$
G_{2}(\overrightarrow{0}, t)=A_{m} e^{-m t}+A_{m^{\prime}} e^{-(m+\Delta m) t},
$$

$$
\begin{aligned}
& G_{3}(\Gamma ; \overrightarrow{0}, t ; \overrightarrow{0}, \tau ; O) \\
& \quad=A_{m} e^{-m t} \ldots\left\{B_{0}+B_{1}\left(e^{-\Delta m \tau}+e^{-\Delta m(t-\tau)}\right)+B_{2} e^{-\Delta m t}\right\} .
\end{aligned}
$$

Now there are only three free parameters for the threepoint correlator due to the transition $m \rightarrow m^{\prime}$ being interchangeable with $m^{\prime} \rightarrow m$ :

$$
B_{0}=\mathrm{FF}(m \rightarrow m, \Gamma, \mathcal{O}),
$$

$$
\begin{aligned}
& B_{1}=\sqrt{\frac{A_{m^{\prime}}}{A_{m}}} \operatorname{FF}\left(m^{\prime} \rightarrow m, \Gamma, \mathcal{O}\right), \\
& B_{2}=\frac{A_{m^{\prime}}}{A_{m}} \operatorname{FF}\left(m^{\prime} \rightarrow m^{\prime}, \Gamma, \mathcal{O}\right) .
\end{aligned}
$$

Note that $B_{2}$ in Eq. (35) can only be extracted if the fit has access to multiple sink times $t$ as only varying the current time $\tau$ cannot distinguish $B_{0}$ from $B_{2}$. 
Since we have access to multiple smearings, we can also construct a combined fit over smearing-dependent $A_{m}$ and $A_{m^{\prime}}$ but a common $m$ and $\Delta m$.

The process for the two-exponential fit is to fit the two-point correlator over a sink time range in which the two-state ansatz is justified. Then we use these extracted parameters in the fit to the three-point correlator using a $\tau$ range that also satisfies a two-state ansatz.

Given the experience in spectroscopy studies, we emphasize that the fit parameter $\Delta m$ should not be taken too literally in terms of the energy gap to the first excited states. The exponential behavior is merely acting to mock up the sum of all excited states over the range of the fit considered. It is for this reason we prefer the nomenclature "two-exponential fit" instead of "two-state fit."

\section{RESULTS}

\section{A. Two-point correlator}

Initial analysis is done on the two-point correlator for the variational analysis, since it is needed for the construction of the combined sink smearing. Via the standard construction below, we can extract the mass assuming a sufficiently large Euclidean time is taken:

$$
\log \left(\frac{G_{2}\left(\Gamma_{4} ; \vec{p}, t\right)}{G_{2}(\Gamma ; \vec{p}, t+\Delta t)}\right) \stackrel{t \gg 0}{\longrightarrow} E_{\vec{p}}^{0} \Delta t .
$$

This function is also graphed for visualization (Fig. 2) with the two-exponential fit function fitted to all source-sink smearing amounts alongside the variational method.

By looking at the mass plots (Fig. 2) we can see that the variational method is producing a correlator similar to the 128 sweeps of the smearing result, but with more excited states being removed. The two-exponential fit seems to indicate that the mass plateau is lower to where you might expect to get a good $\chi_{\mathrm{POF}}^{2}$ for a single state fit in the variational method.

\section{B. Nucleon axial charge $g_{A}$}

The nucleon axial charge has been quite an important benchmark for the validity of lattice QCD calculations. It can be calculated by looking at the operator $\mathcal{O}=i \gamma_{3} \gamma_{5}$

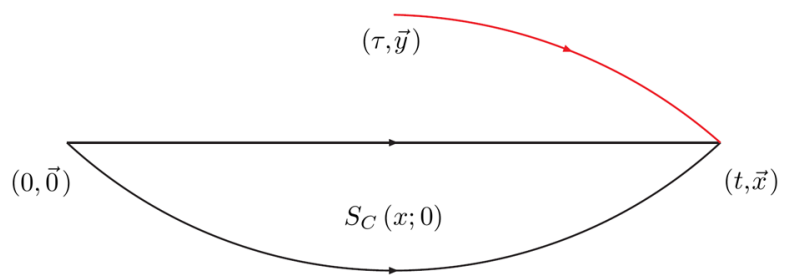

FIG. 1. Diagram showing $\sigma\left(\Gamma ; \vec{p}^{\prime}, t ; y ; 0\right)$ which is the sequential source propagator. $S_{C}(x ; 0)$ is the source used in the inversion, which is represented by the black lines in the diagram.

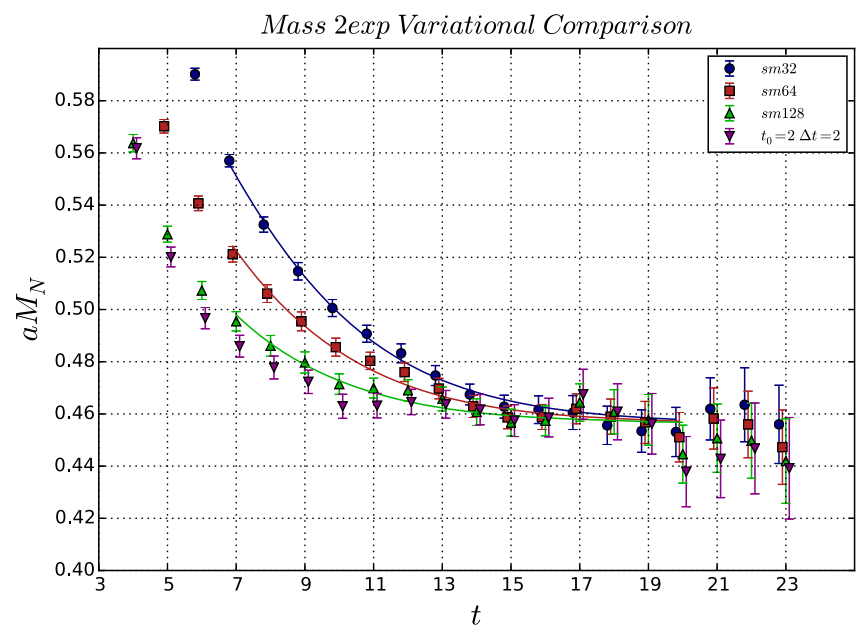

FIG. 2. Mass plots over sink time [using $\Delta t=3$ in Eq. (39)] comparing the different smearings (circle, square, triangle) and the variational method (upside-down triangle). The lines plotted are the two-exponential fit results described in Sec. IV B.

while using a spin projector which corresponds to $\Gamma=\Gamma_{3} \equiv\left(\frac{I+\gamma_{4}}{2}\right) \gamma_{3} \gamma_{5}$.

In Fig. 3, we plot the ratio in Eq. (8) over the current insertion time, using $N_{\text {smear }}=32,64,128$ at both source and sink, along with the variational method, all at a fixed source-sink separation of 13 . For the smeared results, we see that no clear plateau is present around the central current insertion point. In contrast, we can see that the variational method seems to have removed the majority of the contamination from transition matrix elements as it looks to plateau from the current insertion time 5 to 11 .

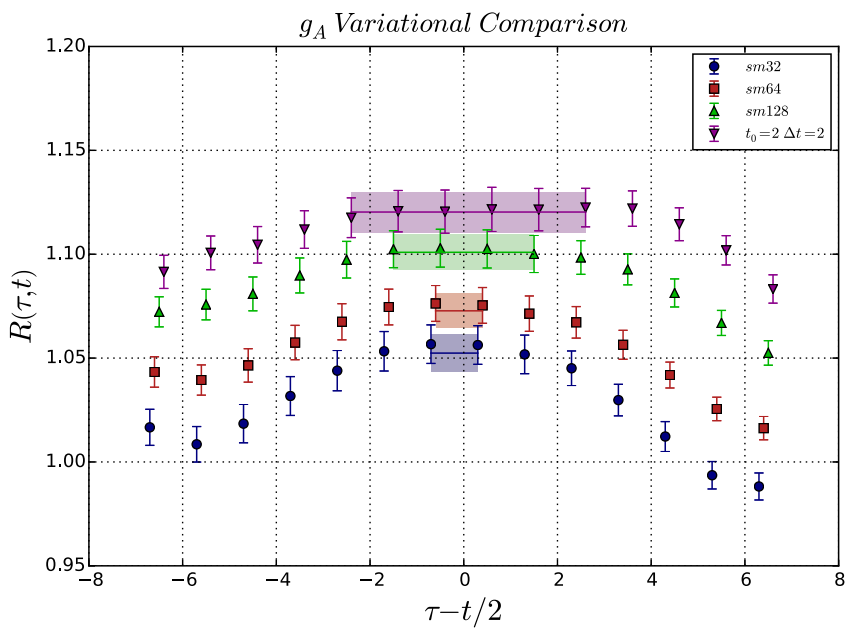

FIG. 3. Graph for $g_{A}$ extracted from the R function defined in Eq. (9). The lines indicate the constant fit value extracted from each set of data used in Fig. 9. All subsequent $\mathrm{R}$ function graphs are plotted over the current insertion time $\tau$ which has been centered about zero via the construction $\tau-t / 2$. This plot compares different diagonally smeared values to the variational method. All results had a source-sink separation of $t=13$. 
Furthermore, the value produced is statistically larger than any of the smeared results, indicating that a poor choice of source and sink operators and/or short source-sink separation times can lead to excited-state contamination which acts to suppress $g_{A}$. This is in agreement with other findings $[15,19]$.

Since we have access to the full $3 \times 3$ correlation matrix at a source-sink separation of 13 , it is possible to utilize any $t_{0}$ and $\Delta t$ calculated in the two-point correlator case. Exploring these parameters in Fig. 4 for the variational results with a source-sink separation of 13 , we see that the variational method parameters $t_{0}$ and $\Delta t$ have minimal effect on the calculation. We choose $t_{0}=2$ and $\Delta t=2$ as it allowed sufficient time after the variational method diagonalization for the correlator to reach the ground state.

In the plots in Fig. 5 we have the summation function defined in Eq. (25) for $g_{A}$ plotted over the source-sink separation times (in which we have summed over the current insertion times). The colors/symbols blue/circle, $\mathrm{red} / \mathrm{square}$, green/triangle and pink/upside-down triangle let us see the change in the line of best fit when we vary $\delta t=0,1,2,3$ respectively in Eq. (25). The top plot shows that the summation fits show no statistically significant change in slope for the different $\delta t$ value results and the line of best fit seems to satisfy the points well to extract a value. Results with small source-sink separations are likely to have the most contamination from higher excitations. They also have smallest statistical error and so can dominate in a weighted fit. By fitting only to the largest three source-sink separated results, we can extend the lines back to compare with the smaller source-sink separated results. Any significant deviation indicates that those smaller source-sink separated results should be excluded from the final fit. For $g_{A}$ in the bottom plot in Fig. 5, we have excluded the two smallest source-sink separated points from the linear fit and

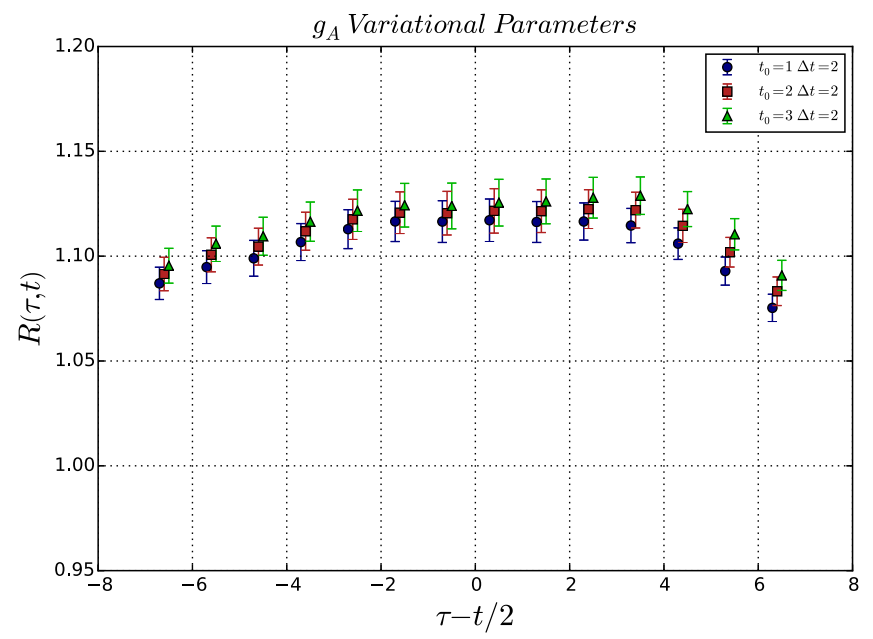

FIG. 4. Graph for $g_{A}$ extracted at each current time $\tau$ comparing all the variational methods produced by varying the $t_{0}$ and $\Delta t$ parameters. This was calculated at a source-sink separation of $t=13$.
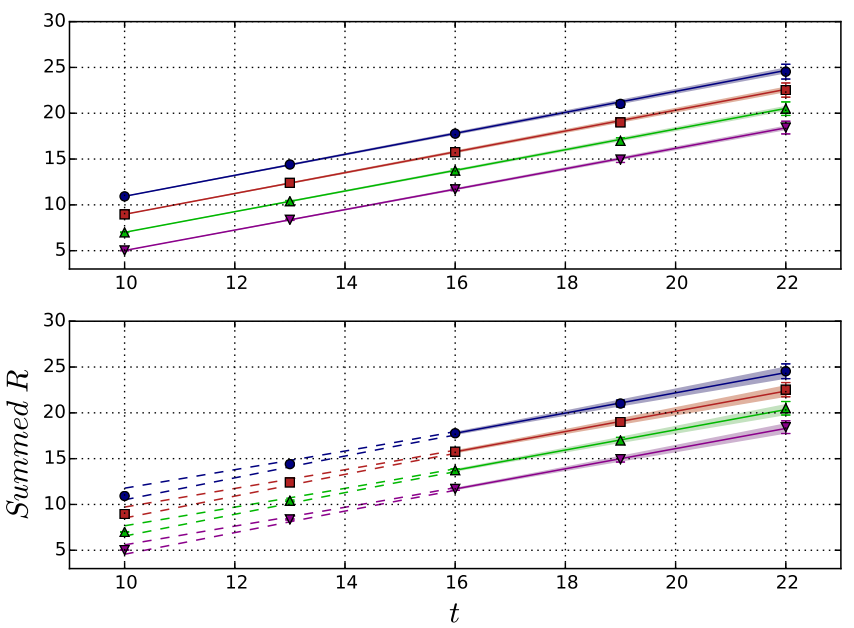

FIG. 5. Summed ratio factor values for multiple source-sink times defined in Eq. (25). The different colors/symbols (blue/ circle, red/square, green/triangle and pink/upside-down triangle respectively) correspond to $\delta t=0,1,2,3$ where $\delta t$ is defined in Eq. (25). The linear line of best fit is used to extract a slope which corresponds to $g_{A}$ and the dashed lines correspond to the projected error ranges for smaller source-sink summed results.

we see that the projected errors do encapsulate the smaller source-sink separated results. We can also see that the errors on the results drastically increase when compared to the top figure, but we see no more $\delta t$ dependence which is required if we are to accept the first order transitional matrix element approximation.

In applying the two-exponential fit to the differently smeared results at a source-sink separation of 13 in Fig. 6 (for $g_{A}$ ), all three smearing fits coincided with one another, having a larger relative error compared to the data points

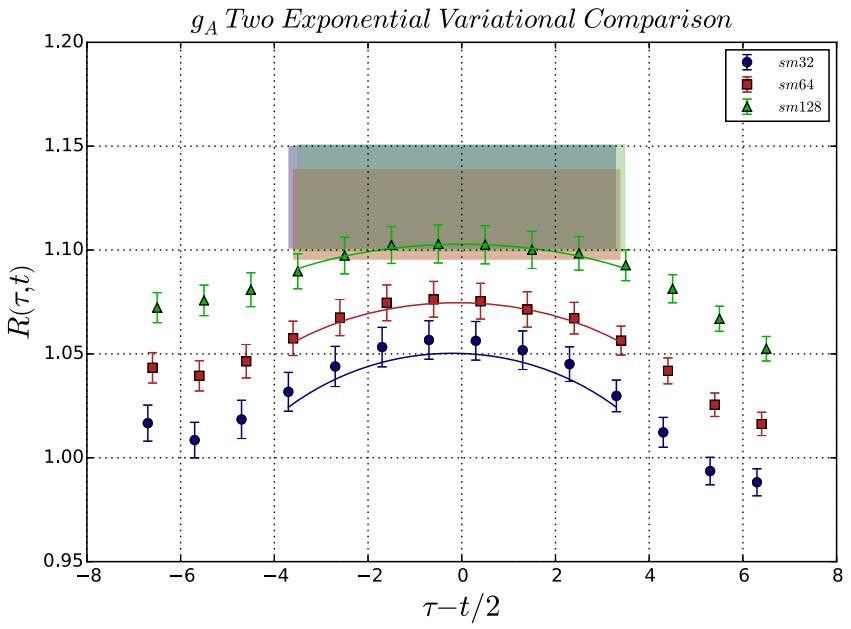

FIG. 6. Graph of Fig. 3 overlaid with a two-exponential fit calculation on each set. The lines are the corresponding fit function and the shaded areas correspond to the $g_{A}$ values extracted from the fit parameters. Since there is no varying in sink time $t$, no $B_{2}$ was calculated in Eq. (35) as discussed at the end of Sec. IV B. 


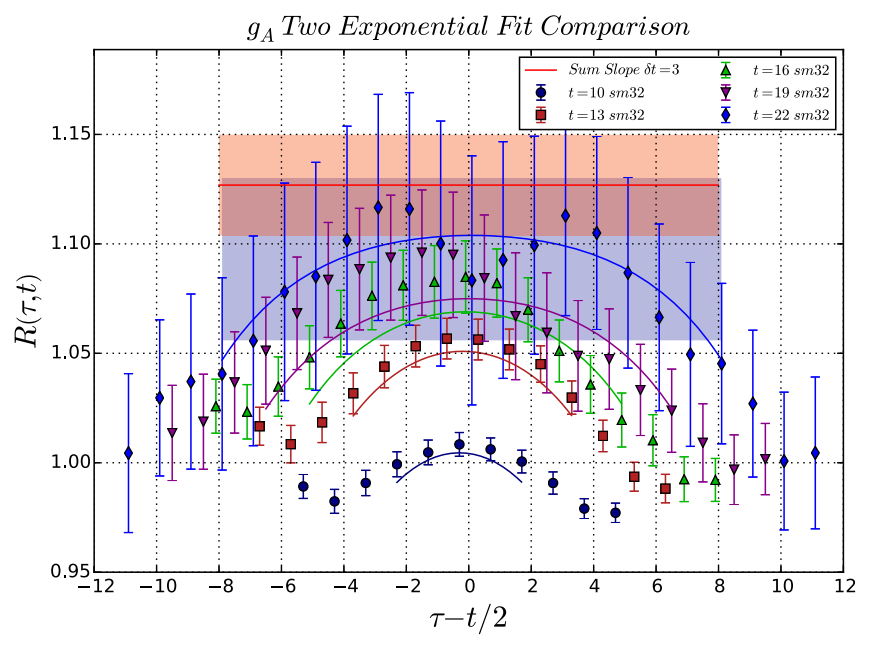

FIG. 7. Graph for $g_{A}$ extracted at each current time $\tau$ for multiple sink times plotted as a comparison to the summation method using $\delta t=3$ (purple line in Fig. 5). Also overlaid with a single two-exponential fit over both current and sink time ( $t$ and $\tau$ ). The lines correspond to the two-exponential fit function constructed and the blue shaded area corresponds to the $g_{A}$ parameter extracted from the two-exponential fit. All results are calculated with 32 multiples of smearing at the source and sink.

they are fitted to and being statistically consistent with a constant fit to the largest smeared (sm128) result.

For $g_{A}$, doing a combined fit to all the source-sink separated data as in Fig. 7 leads to a result that is very similar to a constant fit for the largest source-sink separated result. Similar to the summation method, the two-exponential method is heavily weighted by the smallest source-sink separated values which can be problematic as these values are most susceptible to excited-state contamination.

Figure 8 shows that for the variational method calculation for $g_{A}$, there are no more excited states to remove as the results did not shift up when moving from a source-sink separation of 13 to 16 . Compared to the smallest smeared operators, we see excited states being removed in the change from a source-sink separation of 13 to 16 .

In the final summary plot for $g_{A}$ containing all the extracted values from all the different methods calculated (Fig. 9), we see that the variational method demonstrates reliability and robustness. It produces a value that improves on the results that alter the smearing amounts and small source-sink separated results by removing excited-states. As well as this, it improves on the summation and twoexponential fit method by producing a much more precise result. The variational method result of $g_{A}^{\mathrm{Var}}=1.1203(96)$ agrees within statistical error with the Feynman-Hellmann theorem result of $g_{A}^{\mathrm{FH}}=1.101(24)$ [28] on the same set of gauge field configurations that are used in this work.

\section{Scalar current}

The scalar current form factor has been notorious for its large excited-state contamination. It can be calculated by

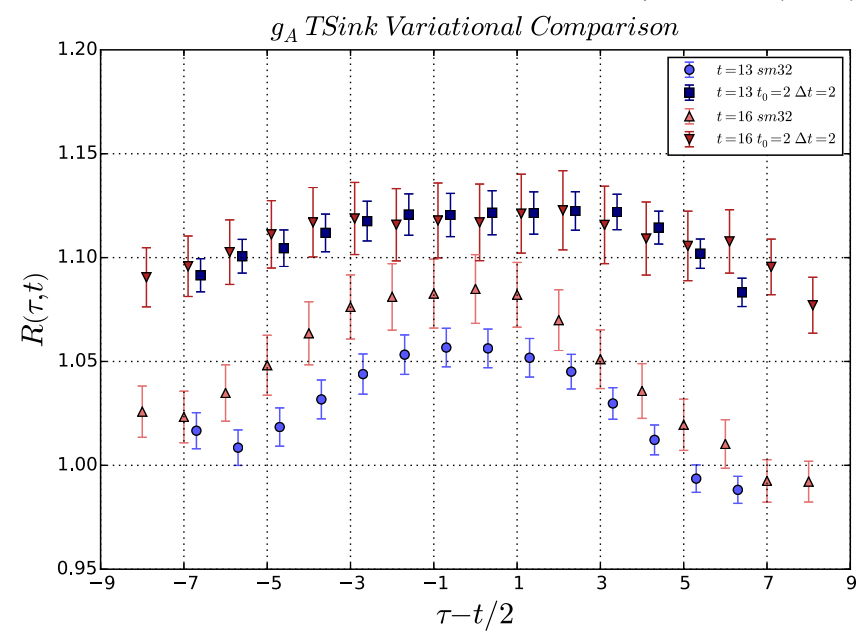

FIG. 8. Graph for $g_{A}$ extracted at each current time $\tau$ comparing different variational results for different source-sink separations (square and upside-down triangle points). This is overlaid with lighter colored results which are the corresponding source-sink separation for the 32 sweeps of smearing results (circle and triangle points).

looking at the operator $\mathcal{O}=I$ while using a spin projector $\Gamma=\Gamma_{4} \equiv \frac{I+\gamma_{4}}{2}$ which corresponds to an unpolarized nucleon. The same analysis can be undertaken for this operator at zero source and sink momentum which leads to a result for the isovector scalar charge, $g_{S}$.

In Fig. 10, we see for $g_{S}$ the variational method producing a flatter ratio as a function of $\tau$ compared to

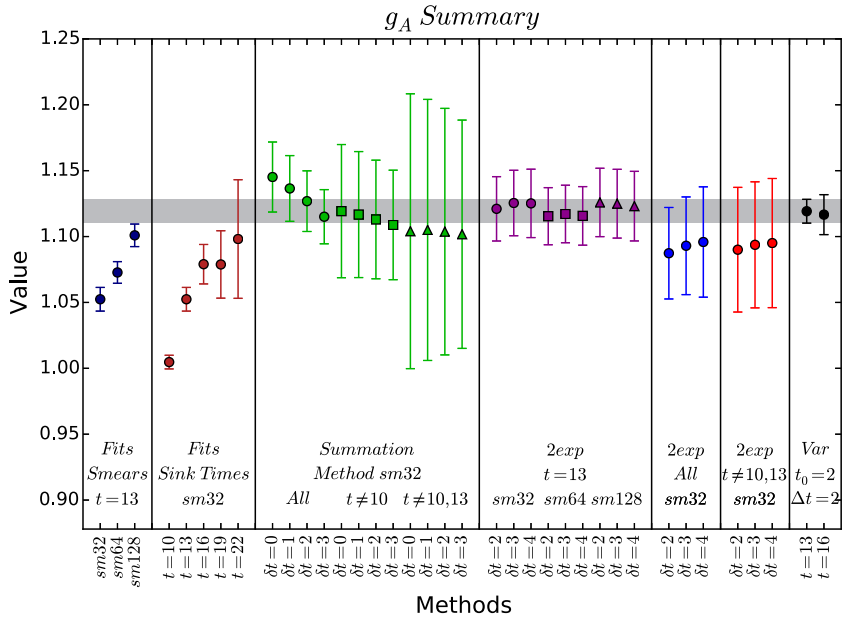

FIG. 9. Summary of all the extracted values for $g_{A}$ over the different methods. "Fits" and "Var" use a constant fit range to extract a value. $\delta t$ is defined in Eq. (25) for the summation method and corresponds to how many points are excluded from the source and sink current times in the two-exponential fit range. "All" refers to using all the source-sink separated results in the analysis and $t \neq n$ refers to excluding $n$ in the calculation. The shaded band helps us to compare the variational method $t=13$ result. 


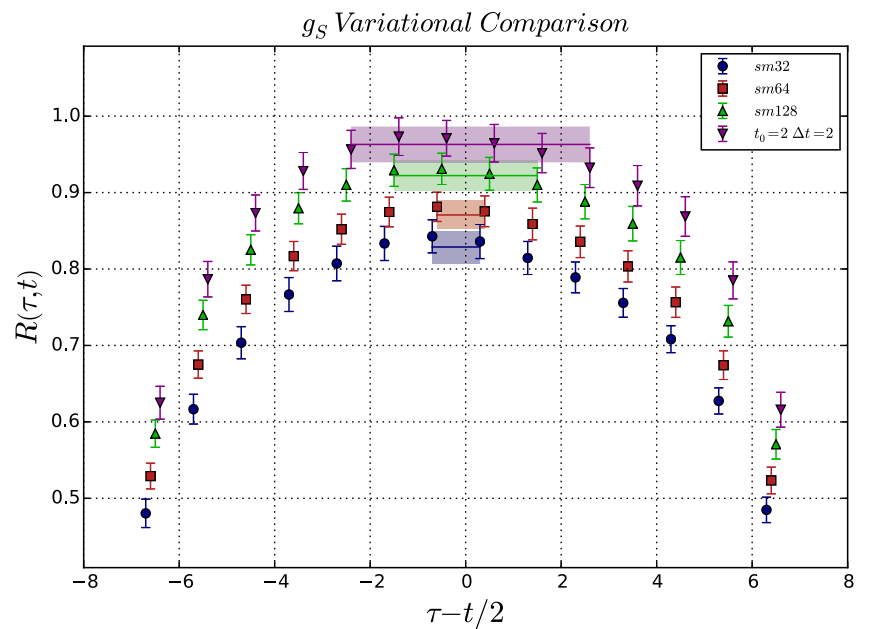

FIG. 10. Graph for $g_{S}$ extracted at each current time $\tau$, comparing different diagonally smeared values to the variational method. The lines indicate the constant fit value extracted from each set of data used in Fig. 15. This was calculated at a sourcesink separation of $t=13$.

the individually smeared correlators. We note that in this case, we see that the transition matrix elements are much larger than $g_{A}$ as there is a larger curvature with respect to the current time insertion $\tau$.

In the summation method results, comparing the four colored slopes passing through the four colors/symbols (blue/circle, red/square, green/triangle and pink/upsidedown triangle respectively) in the top of Fig. 11 shows that the $\delta t=0,1,2,3$ parameter variation is not statistically
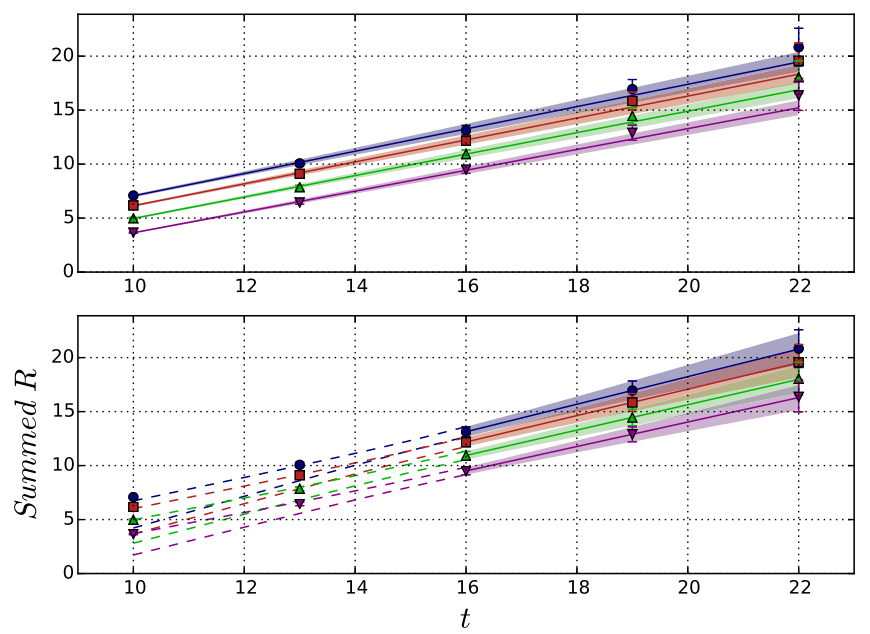

FIG. 11. Summed ratio factor values for multiple source-sink times defined in Eq. (25). The different colors/symbols (blue/ circle, red/square, green/triangle and pink/upside-down triangle respectively) correspond to $\delta t=0,1,2,3$ where $\delta t$ is defined in Eq. (25). The linear line of best fit is used to extract a slope which corresponds to $g_{S}$ and the dashed lines correspond to the projected error ranges for smaller source-sink summed results. significant. However, as the fit is a weighted fit and the smallest source-sink separated points have the smallest errors and the set of points are not linear, the smallest points are forcing the linear function to underestimate the slope of the larger source-sink separated values. Fitting over the larger source-sink separated points in the bottom of Fig. 11 and projecting the fit backwards to smaller times reveals a tension between the results at small and large source-sink separations as the projected errors do not encapsulate the smaller source-sink separated results. This suggests that the error term in Eq. (25) is starting to be statistically significant.

Applying the two-exponential fit to $g_{S}$ for the smeared results in Fig. 12 appears to have made an improvement to all three smeared results. The errors on the parameter extracted have increased compared to the errors associated with the current insertion $\tau$ points.

The two-exponential fit to $g_{S}$ in Fig. 13 again raises a lot of concern over the inclusion of small source-sink separations into the fit. Since the fit is weighted heavily to the smaller source-sink separated results, due to their statistical error the larger source-sink separated results are almost ignored.

Once again for $g_{S}$ in Fig. 14, increasing the source-sink separation for the variational method shows no more statistically significant removal of excited states which cannot be said about the smallest smeared result.

Similarly the summary for $g_{A}, g_{S}$ (Fig. 15) shows that the variational method has removed all excited states and offers far more precise results compared to the summation and two-exponential fit methods. In addition, while not statistically significant, we observe an undesired $\delta t$ dependence for each of the summation method results.

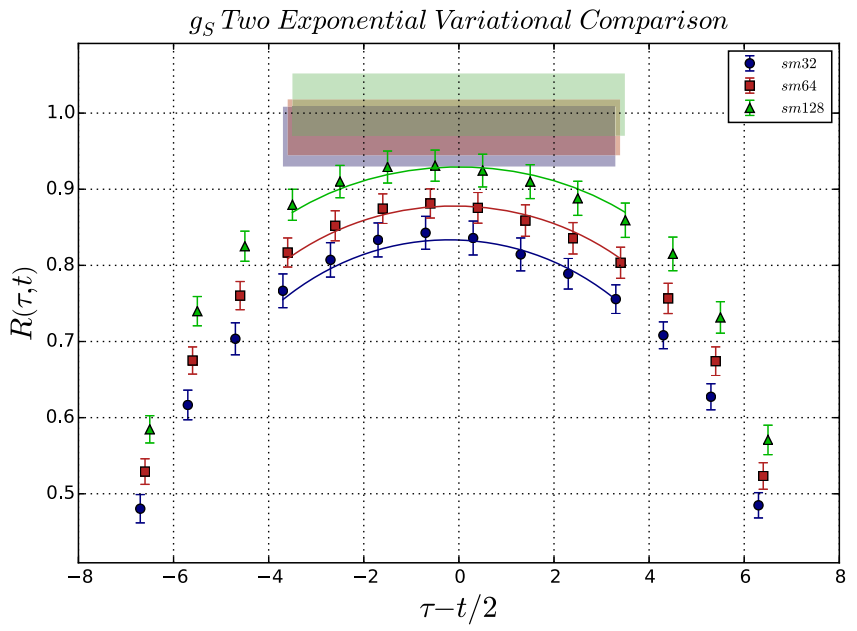

FIG. 12. Graph of Fig. 10 overlaid with a two-exponential fit calculation on each set. The lines are the corresponding fit function and the shaded areas correspond to $g_{S}$ values extracted from the fit parameters. Since there is no varying in sink time $t$, no $B_{2}$ was calculated in Eq. (35) as discussed at the end of Sec. IV B. 


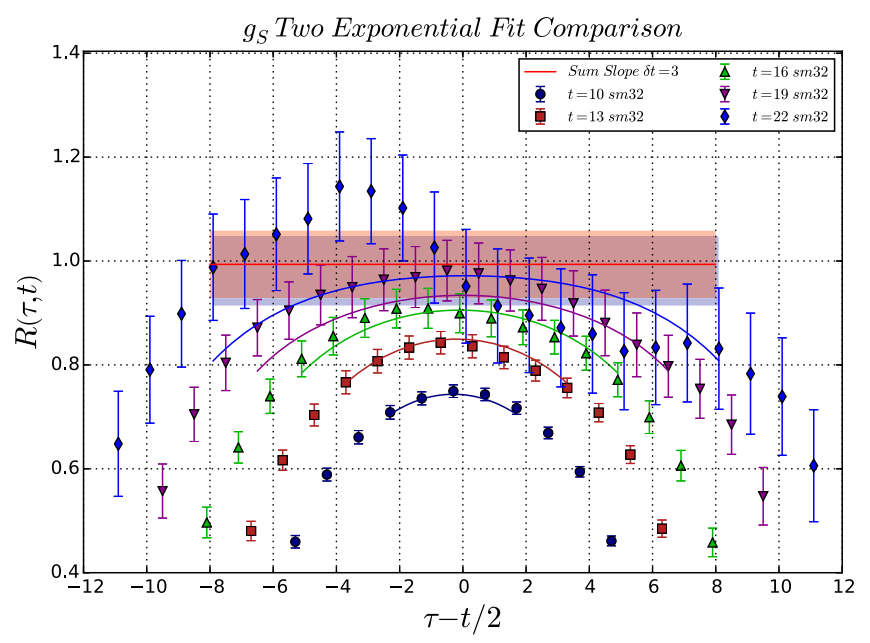

FIG. 13. Graph for $g_{S}$ extracted at each current time $\tau$ for multiple sink times plotted as a comparison to the summation method using $\delta t=3$ (purple line in Fig. 11), also overlaid with a single two-exponential fit over both current and sink time ( $t$ and $\tau$ ). The lines correspond to the two-exponential fit function constructed and the blue shaded area corresponds to the $g_{S}$ parameter extracted from the two-exponential fit. All results are calculated with 32 multiples of smearing at the source and sink.

\section{Quark momentum fraction, $\langle x\rangle$}

Deep inelastic scattering experiments are our primary method for understanding the nucleon and QCD in general. Looking at the operator product expansion, the momentum fractions carried by the quarks and gluons in the nucleon are directly related to the first moment of the structure functions. In any scheme and at any scale, the quark and

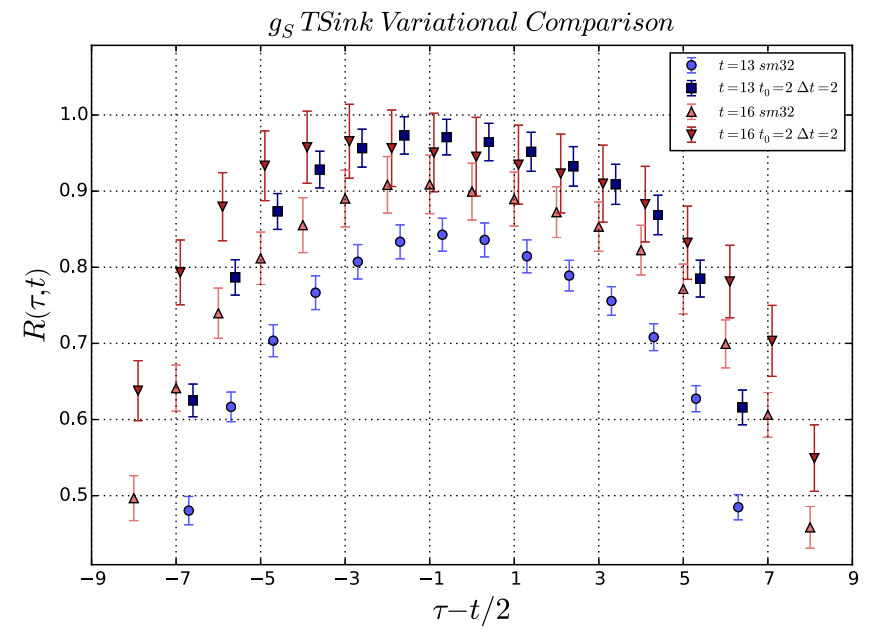

FIG. 14. Graph for $g_{S}$ extracted at each current time $\tau$ comparing different variational results for different source-sink separations (square and upside-down triangle points). This is overlaid with lighter colored results which are the corresponding source-sink separation for the 32 sweeps of smearing results (circle and triangle points).

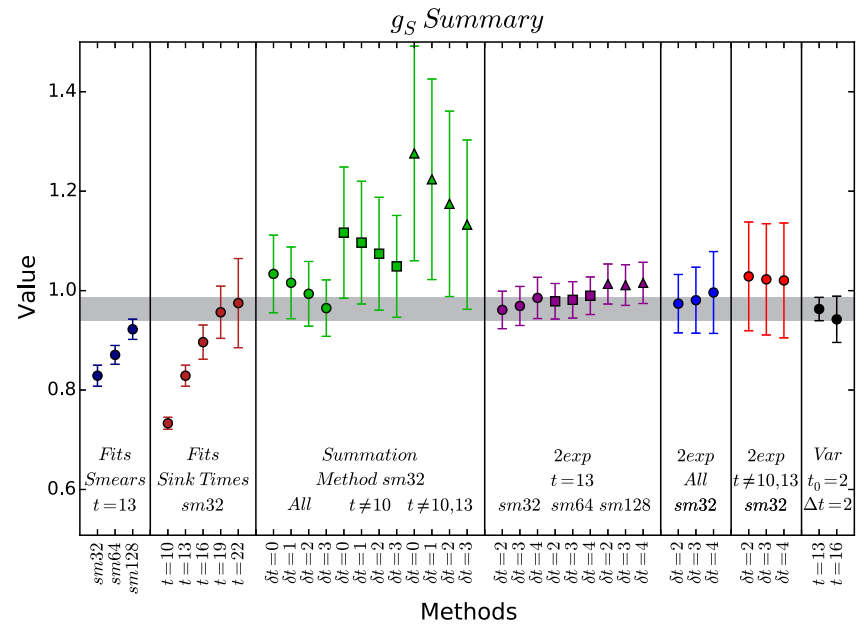

FIG. 15. Summary of all the extracted values for $g_{S}$ over the different methods. "Fits" and "Var" use a constant fit range to extract a value. $\delta t$ is defined in Eq. (25) for the summation method and corresponds to how many points are excluded from the source and sink current times in the two-exponential fit range. "All" refers to using all the source-sink separated results in the analysis and $t \neq n$ refers to excluding $n$ in the calculation. The shaded band helps to compare the variational method $t=13$ result.

gluon momentum fractions sum to unity, providing good motivation for lattice QCD studies.

At the physical quark mass, it is predicted that $\langle x\rangle^{\mathrm{MRST}}=0.157(9)$ [29] whereas the lattice determination of $\langle x\rangle$ at many quark masses has consistently overestimated the quantity over the years. One possible explanation could be due to the contamination from excited states affecting the results.

$\langle x\rangle$ can be calculated by looking at the operator $\mathcal{O}=\gamma_{4} D_{4}-\frac{1}{3}\left(\gamma_{1} D_{1}+\gamma_{2} D_{2}+\gamma_{3} D_{3}\right)$ while using a spin projector which corresponds to $\Gamma=\Gamma_{4}$ as defined in the scalar current results section. The same analysis can be undertaken for this combination. Note that the results presented here are for $R(\tau, t)$ and have not been converted to $\langle x\rangle$ or renormalized. The corresponding conversion required is

$$
\langle x\rangle=\frac{Z_{\langle x\rangle} R(\overrightarrow{0}, \tau ; \overrightarrow{0}, t)}{m^{[0]}}
$$

where $Z_{\langle x\rangle}$ is an unknown renormalization factor for $\langle x\rangle$.

A similar improvement as observed in the previous two quantities has been achieved by the variational method for $\langle x\rangle$ shown in Fig. 16. For this operator we see there is much greater excited-state contamination compared to the precision of the calculation of the current insertion time $\tau$.

Now the summation method fit undertaken in the top of Fig. 17 for $\langle x\rangle$ does show a variation on the $\delta t$ parameter that is statistically significant. We can see for $\langle x\rangle$ the linear 


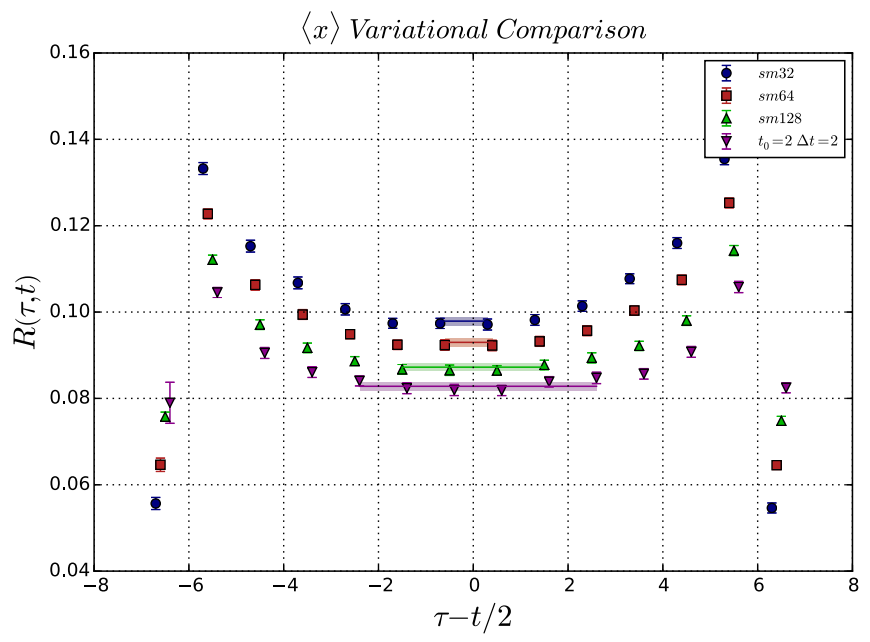

FIG. 16. Graph for $R(\tau, t)$ using the operator for $\langle x\rangle$ extracted at each current time $\tau$, comparing different diagonally smeared values to the variational method. This was calculated at a sourcesink separation of $t=13$.

fit function is not sufficient to approximate the summed $\mathrm{R}$ function values. Again, fitting over larger source-sink separated points in the bottom of Fig. 17 and projecting the errors to smaller times shows that there is an inconsistency as the smaller source-sink separated results do not lie within the fit errors projected to smaller times. This tells us that the two-exponential approximation used in the summation method has broken down.

Applying the two-exponential fit to $\langle x\rangle$ for the smeared results in Fig. 18 looks to have made an improvement to all three smeared results. The errors on the parameter extracted
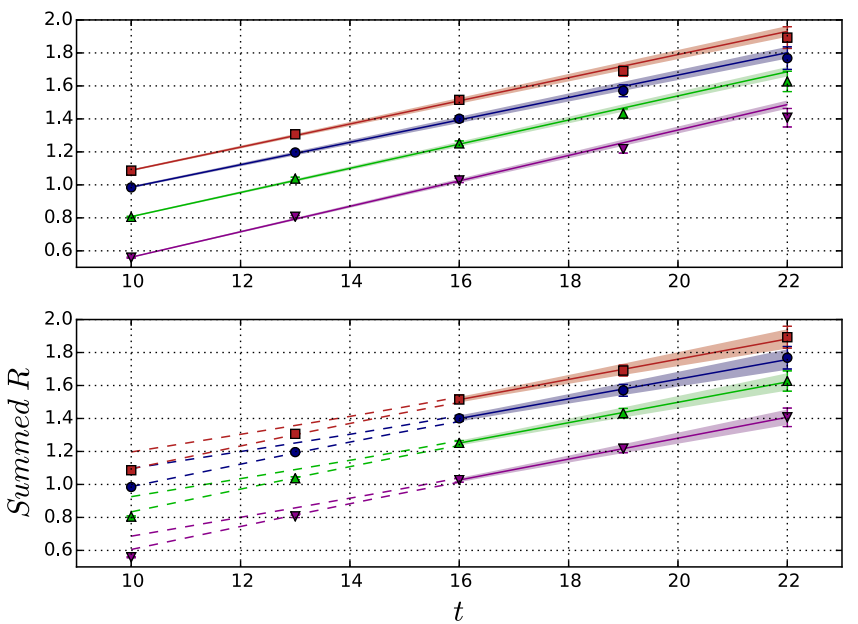

FIG. 17. Summed ratio factor values for multiple source-sink times defined in Eq. (25). The different colors/symbols (blue/ circle, red/square, green/triangle and pink/upside-down triangle respectively) correspond to $\delta t=0,1,2,3$ where $\delta t$ is defined in Eq. (25). The linear line of best fit is used to extract a slope which corresponds to $\langle x\rangle$ and the dashed lines correspond to the projected error ranges for smaller source-sink summed results.

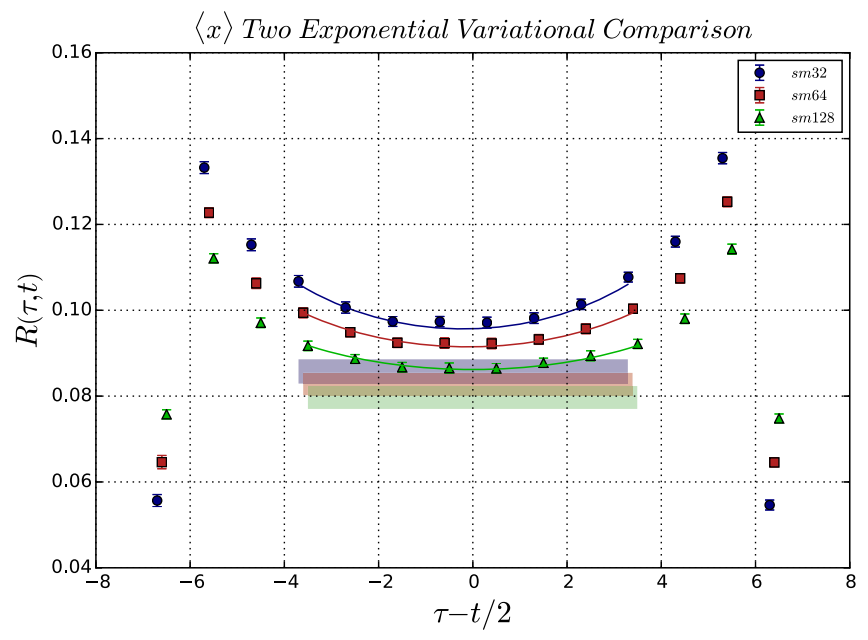

FIG. 18. Graph of Fig. 16 overlaid with a two-exponential fit calculation on each set. The lines are the corresponding fit function and the shaded areas correspond to the $\langle x\rangle$ values extracted from the fit parameters. Since there is no varying in sink time $t$, no $B_{2}$ was calculated in Eq. (35) as discussed at the end of Sec. IV B.

have increased compared to the errors from ratio function points, but for $\langle x\rangle$ it seems that the two-exponential fit was more successful due to the relative size of the excited-state contamination to the precision of the ratio function points.

The two-exponential fit to all five source-sink time separations for $\langle x\rangle$ in Fig. 19 has been more successful relative to the previous two quantities. We see the fit function being approximated appropriately for all current

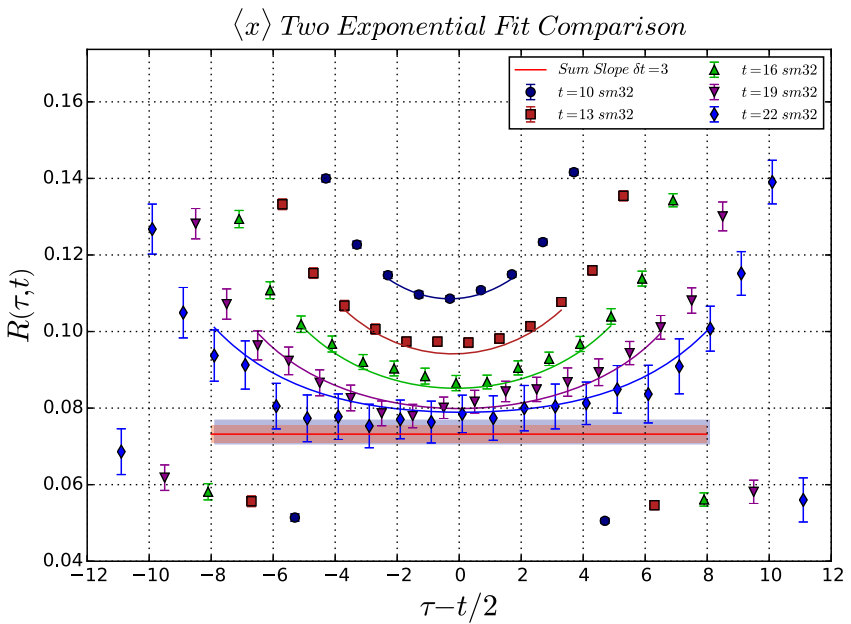

FIG. 19. Graph for $\langle x\rangle$ extracted at each current time $\tau$ for multiple sink times plotted as a comparison to the summation method using $\delta t=3$ (purple line in Fig. 17). Also overlaid with a single two-exponential fit over both current and sink time ( $t$ and $\tau$ ). The lines correspond to the two-exponential fit function constructed and the blue shaded area corresponds to the $\langle x\rangle$ parameter extracted from the two-exponential fit. All results are calculated with 32 multiples of smearing at the source and sink. 


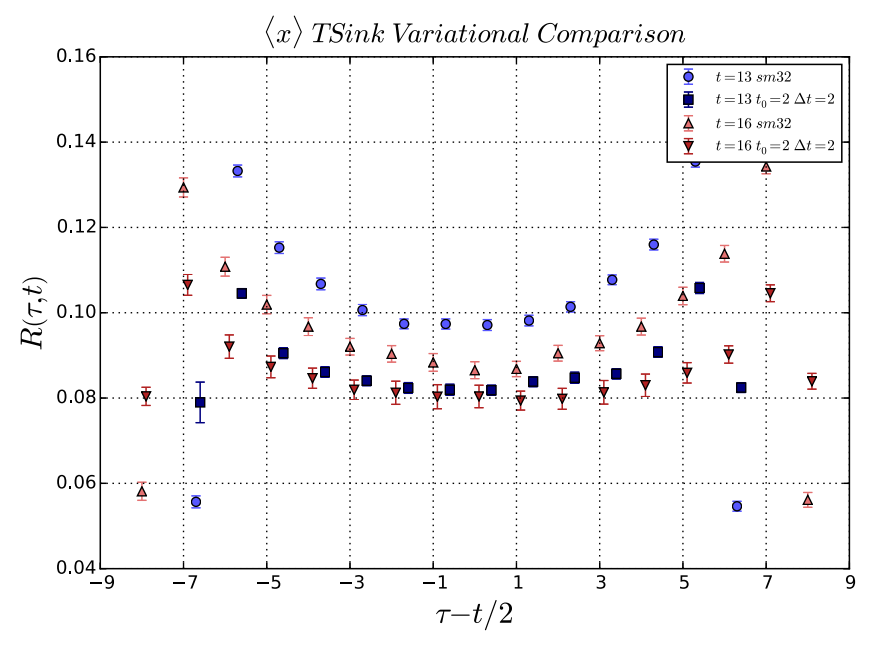

FIG. 20. Graph for $\langle x\rangle$ extracted at each current time $\tau$ comparing different variational results for different source-sink separations (square and upside-down triangle points). This is overlaid with lighter colored results which are the corresponding source-sink separation for the 32 sweeps of smearing results (circle and triangle points).

time and source-sink data sets. But as discussed in the summation method, we must be sure that the twoexponential approximation is satisfied, especially as the excited-state contamination is so large for $\langle x\rangle$.

In the case of $\langle x\rangle$, as displayed in Fig. 20, we see no statistically significant difference between the variational method for the two source-sink separations, which implies that the variational method has dramatically reduced the amount of excited-state contamination. The same cannot be said about the single-smearing analysis.

In summary for $\langle x\rangle$ (Fig. 21) we see that the amount of excited-state contamination removed by the variational method is at the point where the statistical precision has become a larger factor. This puts into question the validity of the summation method and the two-exponential fit results as they show a large disagreement to the variational method. This could be due to insufficient source-sink separated values skewing the results as is indicated by the summation method having a $\delta t$ dependence when it should not. The larger uncertainties due to using very large source-sink separated results could also contribute to the disagreement.

\section{E. Tensor charge $g_{T}$}

Beyond the standard model extensions to neutron beta decay requires tensor charge $g_{T}$ corrections, so the extraction of $g_{T}$ has been of great interest as well. This charge has shown to be less affected by excited-state contamination effects as shown in $[15,19,20]$.

$g_{T}$ can be calculated by looking at the operator $\mathcal{O}=\gamma_{1} \gamma_{2}$ while using a spin projector which corresponds to $\Gamma=$ $\Gamma_{3} \equiv\left(\frac{I+\gamma_{4}}{2}\right) \gamma_{3} \gamma_{5}$.

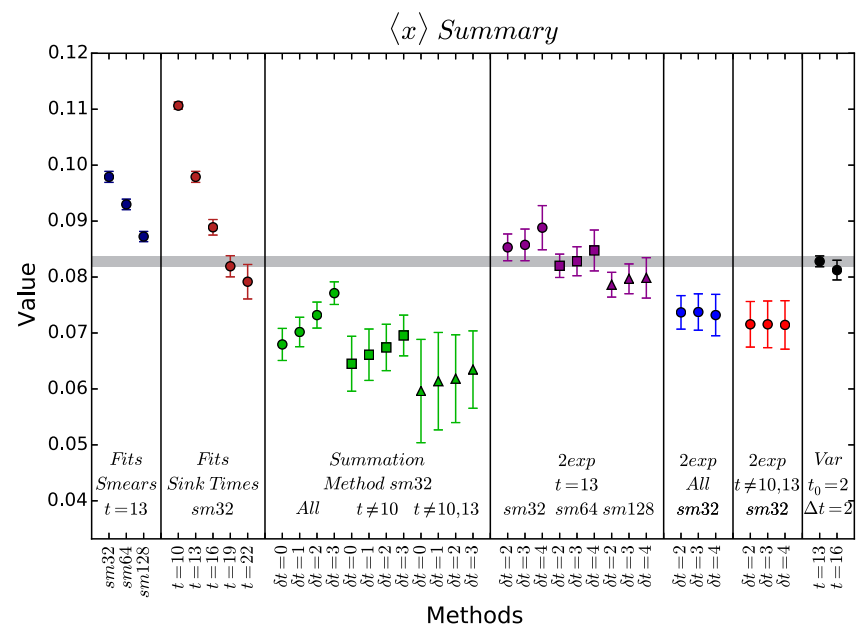

FIG. 21. Summary of all the extracted values for $\langle x\rangle$ over the different methods. "Fits" and "Var" use a constant fit range to extract a value. $\delta t$ is defined in Eq. (25) for the summation method and corresponds to how many points are excluded from the source and sink current times in the two-exponential fit range. "All" refers to using all the source-sink separated results in the analysis and $t \neq n$ refers to excluding $n$ in the calculation. The shaded band helps to compare the variational method $t=13$ result.

In the summary plot in Fig. 22 we also see a discrepancy between the variational method and the two-state fit results. We also see an opposite trend when we vary the level of smearing compared to varying the source-sink separation.

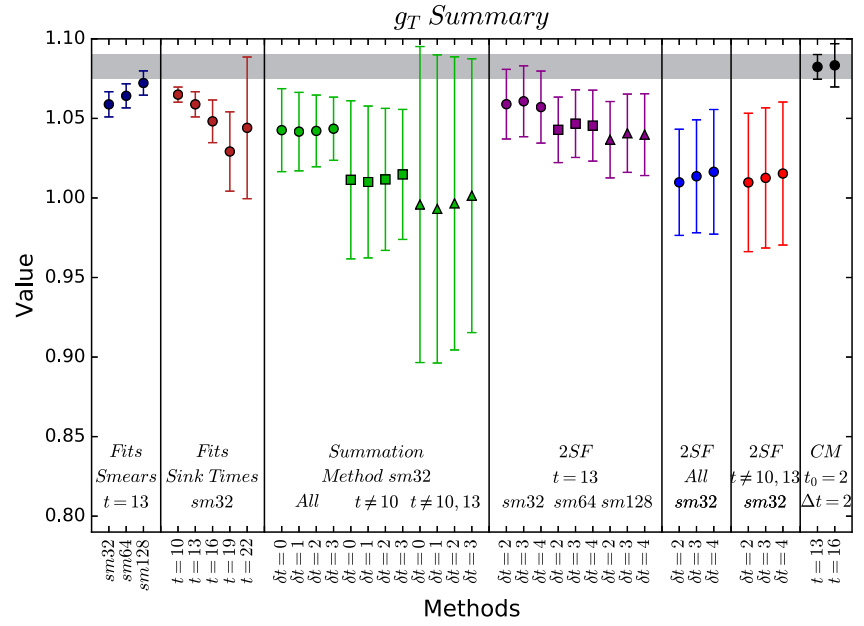

FIG. 22. Summary of all the extracted values for $g_{T}$ over the different methods. "Fits" and "Var" use a constant fit range to extract a value. $\delta t$ is defined in Eq. (25) for the summation method and corresponds to how many points are excluded from the source and sink current times in the two-exponential fit range. "All" refers to using all the source-sink separated results in the analysis and $t \neq n$ refers to excluding $n$ in the calculation. The shaded band helps to compare the variational method $t=13$ result. 
Although the larger source-sink separations do trend away from the variational result, the statistical signal is rapidly degrading. The significance of this trend should therefore be taken with caution.

\section{SUMMARY AND DISCUSSION}

A summary of our results for $g_{A}, g_{S},\langle x\rangle$ and $g_{T}$ presented in the previous section is given in Tables II and III. In the following we summarize our findings.

\section{A. Summation results}

In Figs. 7, 13, and 19, we observe that the summation method looks as if it is improving the result.

TABLE II. Summary of results as displayed in Figs. 9 and 15.

\begin{tabular}{|c|c|c|}
\hline Methods & $g_{A}$ & $g_{S}$ \\
\hline Fit $\mathrm{t}=13, \mathrm{sm} 32$ & $1.0524(90)$ & $0.829(21)$ \\
\hline Fit $\mathrm{t}=13, \mathrm{sm} 64$ & $1.0727(82)$ & $0.871(19)$ \\
\hline Fit $\mathrm{t}=13, \mathrm{sm} 128$ & $1.1009(86)$ & $0.922(20)$ \\
\hline Fit $\mathrm{t}=10, \mathrm{sm} 32$ & $1.0047(52)$ & $0.733(12)$ \\
\hline Fit $\mathrm{t}=13, \mathrm{sm} 32$ & $1.0524(90)$ & $0.829(21)$ \\
\hline Fit $\mathrm{t}=16, \mathrm{sm} 32$ & $1.079(15)$ & $0.896(34)$ \\
\hline Fit $\mathrm{t}=19, \mathrm{sm} 32$ & $1.079(26)$ & $0.956(53)$ \\
\hline Fit $\mathrm{t}=22, \mathrm{sm} 32$ & $1.098(45)$ & $0.975(90)$ \\
\hline Sum All $\delta \mathrm{t}=0$ & $1.145(27)$ & $1.034(78)$ \\
\hline Sum All $\delta \mathrm{t}=1$ & $1.136(25)$ & $1.016(72)$ \\
\hline Sum All $\delta \mathrm{t}=2$ & $1.127(23)$ & $0.994(65)$ \\
\hline Sum All $\delta \mathrm{t}=3$ & $1.115(21)$ & $0.965(57)$ \\
\hline Sum $\mathrm{t} \neq 10 \delta \mathrm{t}=0$ & $1.119(51)$ & $1.12(13)$ \\
\hline Sum $t \neq 10 \delta \mathrm{t}=1$ & $1.117(48)$ & $1.10(12)$ \\
\hline Sum $\mathrm{t} \neq 10 \delta \mathrm{t}=2$ & $1.113(45)$ & $1.07(11)$ \\
\hline Sum $\mathrm{t} \neq 10 \delta \mathrm{t}=3$ & $1.109(42)$ & $1.05(10)$ \\
\hline Sum $t \neq 10,13 \delta \mathrm{t}=0$ & $1.10(10)$ & $1.28(22)$ \\
\hline Sum $t \neq 10,13 \delta \mathrm{t}=1$ & $1.105(99)$ & $1.22(20)$ \\
\hline Sum $t \neq 10,13 \delta \mathrm{t}=2$ & $1.104(94)$ & $1.17(19)$ \\
\hline Sum $t \neq 10,13 \delta \mathrm{t}=3$ & $1.102(87)$ & $1.13(17)$ \\
\hline $2 \exp \mathrm{t}=13, \operatorname{sm} 32 \delta \mathrm{t}=2$ & $1.121(24)$ & $0.961(38)$ \\
\hline $2 \exp \mathrm{t}=13, \operatorname{sm} 32 \delta \mathrm{t}=3$ & $1.125(25)$ & $0.969(39)$ \\
\hline $2 \exp \mathrm{t}=13, \operatorname{sm} 32 \delta \mathrm{t}=4$ & $1.125(26)$ & $0.985(41)$ \\
\hline $2 \exp \mathrm{t}=13, \operatorname{sm} 64 \delta \mathrm{t}=2$ & $1.115(22)$ & $0.979(36)$ \\
\hline $2 \exp \mathrm{t}=13, \operatorname{sm} 64 \delta \mathrm{t}=3$ & $1.117(22)$ & $0.981(37)$ \\
\hline $2 \operatorname{exp~t}=13, \operatorname{sm} 64 \delta \mathrm{t}=4$ & $1.116(22)$ & $0.990(38)$ \\
\hline $2 \exp \mathrm{t}=13, \operatorname{sm} 128 \delta \mathrm{t}=2$ & $1.126(26)$ & $1.013(40)$ \\
\hline $2 \operatorname{exp~} \mathrm{t}=13, \operatorname{sm} 128 \delta \mathrm{t}=3$ & $1.125(26)$ & $1.011(41)$ \\
\hline $2 \operatorname{exp~} \mathrm{t}=13, \operatorname{sm} 128 \delta \mathrm{t}=4$ & $1.123(26)$ & $1.015(41)$ \\
\hline $2 \exp$ All $\operatorname{sm} 32 \delta \mathrm{t}=2$ & $1.087(35)$ & $0.974(59)$ \\
\hline $2 \exp$ All $\operatorname{sm} 32 \delta \mathrm{t}=3$ & $1.093(37)$ & $0.981(66)$ \\
\hline $2 \exp$ All $\operatorname{sm} 32 \delta \mathrm{t}=4$ & $1.096(42)$ & $0.996(82)$ \\
\hline $2 \exp \mathrm{t} \neq 10,13 \operatorname{sm} 32 \delta \mathrm{t}=2$ & $1.090(47)$ & $1.03(11)$ \\
\hline $2 \exp \mathrm{t} \neq 10,13 \operatorname{sm} 32 \delta \mathrm{t}=3$ & $1.094(48)$ & $1.02(11)$ \\
\hline $2 \exp \mathrm{t} \neq 10,13 \operatorname{sm} 32 \delta \mathrm{t}=4$ & $1.095(49)$ & $1.02(12)$ \\
\hline $\operatorname{Var} \mathrm{t}=13, t_{0}=2 \Delta \mathrm{t}=2$ & $1.1203(95)$ & $0.963(23)$ \\
\hline $\operatorname{Var} \mathrm{t}=16, t_{0}=2 \Delta \mathrm{t}=2$ & $1.118(16)$ & $0.942(47)$ \\
\hline
\end{tabular}

However, when looking at $g_{S}$ and $\mathrm{R}$ for $\langle x\rangle$ extracted values in their respective summary plots (Figs. 15 and 21) we can see a dependence in the $\delta t$ value when, if our two-exponential ansatz were satisfied, it should have no or minimal effect.

This is seen more clearly when considering summation fits excluding smaller source-sink separations $\left(g_{A}\right.$ in Fig. 5, $g_{S}$ in Fig. 11 and $\langle x\rangle$ in Fig. 17). When we exclude the smaller source-sink separated results, we can see that the two-exponential ansatz is breaking down for $g_{S}$ and $\langle x\rangle$ as the data points do not lie within the errors projected to earlier source-sink separated time values.

TABLE III. Summary of results as displayed in Figs. 21-22. To obtain $\langle x\rangle$, one must divide out the mass of the proton and then apply the renormalization constant to $\mathrm{R}$.

\begin{tabular}{|c|c|c|}
\hline Methods & $\mathrm{R}$ for $\langle x\rangle$ & $g_{T}$ \\
\hline Fit $\mathrm{t}=13, \mathrm{sm} 32$ & $0.09790(98)$ & $1.0589(79)$ \\
\hline Fit $\mathrm{t}=13, \mathrm{sm} 64$ & $0.09298(94)$ & $1.0642(76)$ \\
\hline Fit $\mathrm{t}=13, \operatorname{sm} 128$ & $0.08724(91)$ & $1.0722(76)$ \\
\hline Fit $\mathrm{t}=10, \mathrm{sm} 32$ & $0.08724(91)$ & $1.0650(47)$ \\
\hline Fit $\mathrm{t}=13, \mathrm{sm} 32$ & $0.09790(98)$ & $1.0589(79)$ \\
\hline Fit $\mathrm{t}=16, \mathrm{sm} 32$ & $0.0889(14)$ & $1.048(13)$ \\
\hline Fit $\mathrm{t}=19, \mathrm{sm} 32$ & $0.0819(19)$ & $1.029(25)$ \\
\hline Fit $\mathrm{t}=22, \mathrm{sm} 32$ & $0.0792(31)$ & $1.044(45)$ \\
\hline Sum All $\delta \mathrm{t}=0$ & $0.0680(28)$ & $1.043(26)$ \\
\hline Sum All $\delta \mathrm{t}=1$ & $0.0702(26)$ & $1.042(25)$ \\
\hline Sum All $\delta \mathrm{t}=2$ & $0.0732(23)$ & $1.042(23)$ \\
\hline Sum All $\delta \mathrm{t}=3$ & $0.0771(20)$ & $1.044(20)$ \\
\hline Sum $\mathrm{t} \neq 10 \delta \mathrm{t}=0$ & $0.0645(49)$ & $1.011(50)$ \\
\hline Sum $\mathrm{t} \neq 10 \delta \mathrm{t}=1$ & $0.0661(46)$ & $1.010(48)$ \\
\hline Sum $\mathrm{t} \neq 10 \delta \mathrm{t}=2$ & $0.0674(41)$ & $1.012(45)$ \\
\hline Sum $\mathrm{t} \neq 10 \delta \mathrm{t}=3$ & $0.0696(36)$ & $1.015(41)$ \\
\hline Sum $\mathrm{t} \neq 10,13 \delta \mathrm{t}=0$ & $0.0596(92)$ & $0.996(99)$ \\
\hline Sum $t \neq 10,13 \delta \mathrm{t}=1$ & $0.0614(87)$ & $0.993(97)$ \\
\hline Sum $\mathrm{t} \neq 10,13 \delta \mathrm{t}=2$ & $0.0614(87)$ & $0.997(92)$ \\
\hline Sum $t \neq 10,13 \delta \mathrm{t}=3$ & $0.0635(69)$ & $1.001(86)$ \\
\hline $2 \exp \mathrm{t}=13, \operatorname{sm} 32 \delta \mathrm{t}=2$ & $0.0853(23)$ & $1.059(22)$ \\
\hline $2 \exp \mathrm{t}=13, \operatorname{sm} 32 \delta \mathrm{t}=3$ & $0.0857(28)$ & $1.061(22)$ \\
\hline $2 \exp \mathrm{t}=13, \operatorname{sm} 32 \delta \mathrm{t}=4$ & $0.0888(39)$ & $1.057(23)$ \\
\hline $2 \exp \mathrm{t}=13, \operatorname{sm} 64 \delta \mathrm{t}=2$ & $0.0820(21)$ & $1.043(21)$ \\
\hline $2 \exp \mathrm{t}=13, \operatorname{sm} 64 \delta \mathrm{t}=3$ & $0.0828(26)$ & $1.047(21)$ \\
\hline $2 \exp \mathrm{t}=13, \operatorname{sm} 64 \delta \mathrm{t}=4$ & $0.0848(37)$ & $1.045(22)$ \\
\hline $2 \operatorname{exp~} \mathrm{t}=13, \operatorname{sm} 128 \delta \mathrm{t}=2$ & $0.0786(22)$ & $1.037(24)$ \\
\hline $2 \operatorname{exp~} \mathrm{t}=13, \operatorname{sm} 128 \delta \mathrm{t}=3$ & $0.0797(27)$ & $1.041(25)$ \\
\hline $2 \operatorname{exp~} \mathrm{t}=13, \operatorname{sm} 128 \delta \mathrm{t}=4$ & $0.0799(36)$ & $1.040(26)$ \\
\hline $2 \exp$ All $\operatorname{sm} 32 \delta \mathrm{t}=2$ & $0.0737(30)$ & $1.010(33)$ \\
\hline $2 \exp$ All $\operatorname{sm} 32 \delta \mathrm{t}=3$ & $0.0737(33)$ & $1.014(35)$ \\
\hline $2 \exp$ All $\operatorname{sm} 32 \delta \mathrm{t}=4$ & $0.0732(37)$ & $1.016(39)$ \\
\hline $2 \exp t \neq 10,13 \operatorname{sm} 32 \delta \mathrm{t}=2$ & $0.0716(41)$ & $1.010(43)$ \\
\hline $2 \exp t \neq 10,13 \operatorname{sm} 32 \delta \mathrm{t}=3$ & $0.0715(42)$ & $1.013(44)$ \\
\hline $2 \exp \mathrm{t} \neq 10,13 \operatorname{sm} 32 \delta \mathrm{t}=4$ & $0.0714(43)$ & $1.015(45)$ \\
\hline $\operatorname{Var} \mathrm{t}=13, t_{0}=2 \Delta \mathrm{t}=2$ & $0.08281(97)$ & $1.0824(78)$ \\
\hline $\operatorname{Var} \mathrm{t}=16, t_{0}=2 \Delta \mathrm{t}=2$ & $0.0812(18)$ & $1.083(14)$ \\
\hline
\end{tabular}




\section{B. Two-exponential fit results}

The graphs called "Two Exponential Variational Comparison" seem to show minimal improvement for $g_{A}$ (Fig. 6), some improvements for $g_{S}$ (Fig. 12) and the most improvement for $\langle x\rangle$ (Fig. 18). Poor determination would be attributed to not being able to distinguish excited-state contamination from our error within a fit range in which a two-exponential ansatz is justified. These results give a good demonstration of using fitting functions to remove transitional matrix elements. In all cases, the smaller smeared results (with larger excited-state contamination) extract a value closer to the larger smeared results. From the summary plots (Figs. 9, 15, and 21), we see minimal effect on the $\delta t$ fit parameter for the two-exponential fit method.

Extending to the full source-sink separated set of results in the graphs called "Two Exponential Fit Comparison" for 32 sweeps of smearing (Figs. 7, 13, and 19), we see that the fit is weighted predominately by the smallest source-sink separations. Furthermore, we see how poorly the larger source-sink separated results are in terms of symmetry about the middle current insertion time, as well as deformations to the expected curved fit lines. Although using the two-exponential fit method controls the excited states better than using a single source-sink separation, we found there was no improvement to a constant fit over the largest source-sink separation for $g_{A}$ and $g_{S}$ and a questionable improvement for $\langle x\rangle$.

\section{Variational results}

Beginning with the effective mass plots in Fig. 2 where the effective masses for the three different smearing results were compared to the variational method, the variational method allows us to extract the mass from the two-point correlator beginning from an earlier time slice compared to the individually smeared results. The improvement is due to the excited states being suppressed when constructing the optimal correlator in Eq. (13).

In Figs. 3, 10, and 16 we compare the ratio functions [Eq. (8)] for the three different smearing results to the variational method in which the functions are varied over the current insertion time $\tau$ for a fixed source-sink separation $t=13$. The figures show how applying the variational method improves the suppression of excited-state contamination. The ability to fit a plateau over a much larger current insertion time $\tau$ shows how the transition matrix elements are being sufficiently suppressed compared to the individually smeared results. The shift in each of the ratio values for each particular $\tau$ shows how the variational method is suppressing all types of excited-state contamination ("transition" and "excited-state to excitedstate" matrix elements).

The final collection of graphs, called "TSink Variational Comparison" (Figs. 8, 14, and 20), compares the variational method to the 32 sweeps of smearing results over the current insertion times $\tau$ and the source-sink separation of
13 and 16. All three quantities calculated with the variational method show no statistically significant difference between the two source-sink separations. This shows us that choosing a source-sink separation of 16 for the variational method gives us a result where the residual excited-state contamination is smaller than the statistical error. Compared to the tinted points (circle and triangle points), a much larger source-sink separation in the 32 sweeps of the smearing case is needed to remove the remaining excited-state contamination.

\section{Findings}

We can see that in all the values analyzed, the variational method improved our result while only sacrificing minimal uncertainty. Varying the variational parameters proved to be irrelevant as all variations were consistent with each other.

In contrast, the summation and two-exponential fit methods either fell short of removing the excited-state contamination or required the inclusion of source-sink time separations that induced large uncertainties in the results. Also, careful consideration must be taken to the twoexponential ansatz in both methods, as using insufficient source-sink separations might not satisfy the ansatz for any of the current insertion times. The two-exponential fit will improve as you improve the statistics of the calculation, as you will be able to distinguish the ground and excited states better from the uncertainties on the values. A possible improvement might be to weight the larger source-sink separated results with more statistics over the shorter source-sink separated results.

\section{E. Cost/benefit analysis}

Assuming we have an equal number of gauge fields for our particular $\kappa$ value (or pion mass), we can model the efficiency as to how many inversions we undertake per gauge field. One inversion is required for calculating the two-point correlator, and then a second inversion is required for each specific three-point correlator we want to calculate. The fixed sink method requires that we choose a sink time, sink momentum, and spin projector and choose which quark the current acts on for a fixed hadron before the three-point correlator is calculated.

The variational method requires $n_{\text {basis }}$ inversions to create the two-point correlators, where $n_{\text {basis }}$ is the number of basis interpolating fields used (e.g. three smearings for this work). Then a further $n_{\text {basis }}$ is required to create a particular fixed sink resulting correlator as shown in Sec. III B.

The two-exponential fit and summation methods are identical to the standard way, but they create $n_{t}$ multiples of the three-point correlator, where $n_{t}$ is the number of sourcesink time separations.

For this analysis, simulations were performed with zero sink momentum and two different spin projectors for both up and down quark contributions to the proton. This results 
TABLE IV. Comparative computational times for each method for two-point and three-point correlators as well as the total. $n_{t}$ is the number of source-sink time separations used and $n_{\text {basis }}$ is the number of basis interpolating fields used in the variational method (Var).

\begin{tabular}{lccc}
\hline \hline Create & Standard & $\begin{array}{c}\text { 2exp and SM } \\
\left(\text { over } n_{t}\right)\end{array}$ & $\begin{array}{c}\text { Var } \\
\left.\text { (over } n_{\text {basis }}\right)\end{array}$ \\
\hline$C_{2}$ & 1 & 1 & $n_{\text {basis }}$ \\
$C_{3}$ & 4 & $4 n_{t}$ & $4 n_{\text {basis }}$ \\
Total & 5 & $1+4 n_{t}$ & $5 n_{\text {basis }}$ \\
This paper & 5 & 21 & 15 \\
\hline \hline
\end{tabular}

in four times the number of inversions for each three-point correlator required. The inversion numbers are outlined in Table IV.

\section{CONCLUSION}

In lattice simulations of three-point correlation functions it is most common to make use of a sequential inversion "through the sink." This allows the efficient study of many operators and choices of momentum transfer for essentially fixed computational cost. To gain control of statistical uncertainties, it is preferable to keep the source-sink separation time short. Unfortunately, aggressive choices of source-sink separations lead to significant contamination from excited states. One can extend the source-sink separation, yet for fixed computational cost, the results presented here suggest that by the time the excited-state contamination is under control the statistical signal is almost lost. This motivates the study of competing techniques which have been proposed to mitigate the excitedstate contamination problem.

Theoretically, the summation method offers a parametric suppression of excited-state contamination. Nevertheless, in similar fashion to the plateau method, we find this technique to be plagued by the difficulty of identifying the shortest source-sink separation which can reliably be used in a given fit. The high statistical precision obtained at short source-sink separated times can potentially lead to a significant distortion of the fit and result in erroneous extraction of matrix elements.

The two-exponential fit allows the influence of excitedstate contamination to be accounted for numerically. The analysis presented here suggests that this technique offers an improved determination of the desired matrix elements. The method appears rather robust with respect to modified fit ranges, which might indicate that the two exponentials are sufficient to model the two states of the correlators. The uncertainty estimate appears reliable in general, yet caution should be taken if the extracted value lies outside the fit at the largest source-sink time separation.
In contrast to the two previous techniques, which require investigation of an extended range of source-sink separated correlators, the variational approach is designed to reduce the excited-state contamination at early times where the statistical signal is still strong. We find that we were reliably able to apply a plateau fit to the variational method calculation due to obtaining a larger number of current insertion time results that had plateaued to a common value. This indicates that all transition matrix elements were sufficiently suppressed with respect to the uncertainties. Although we knew that all excited-state contamination effects should be suppressed from examining the effective mass plots [Eq. (2)], having a larger source-sink separated result for the variational method confirmed our initial choice of source-sink time separation.

We anticipate that the results presented here will be naturally applicable to a more general set of observables. In particular, at finite momentum transfer the variational approach can be easily adapted to allow for momentumdependent operator projection at the source. Although a priori knowledge of a semioptimal smearing for zero momentum operator projection at the source and sink may be sufficient for these types of calculations, moving to momentum-dependant operator projection at the source may have different optimal smearings for each source momentum calculated. Results will be presented in a future publication.

While the results presented here are just for a single quark mass, the issue of excited-state contamination is anticipated to become even more prevalent at light quark masses and large volumes. Given that statistical fluctuations are also greater at light quark masses, there will be increasing demand for techniques which are robust at short source-sink separations, such as the variational method described here.

\section{ACKNOWLEDGMENTS}

The generation of the numerical configurations was performed using the BQCD lattice QCD program [30] on the IBM BlueGeneQ using DIRAC 2 resources (EPCC, Edinburgh, UK); the BlueGene P and Q at NIC (Jülich, Germany); and the Cray XC30 at HLRN (the North-German Supercomputing Alliance). Some of the simulations were undertaken on the NCI National Facility in Canberra, Australia, which is supported by the Australian Commonwealth Government. We also acknowledge the Phoenix cluster at the University of Adelaide. The BlueGene codes were optimized using Bagel [31]. This investigation has been supported in part by the Australian Research Council under Grants No. FT120100821, No. FT100100005, No. DP150103164, No. DP140103067 and No. CE110001004. 
[1] G. P. Engel, C. B. Lang, M. Limmer, D. Mohler, and A. Schäfer (Bern-Graz-Regensburg Collaboration), Phys. Rev. D 82, 034505 (2010).

[2] R. G. Edwards, J. J. Dudek, D. G. Richards, and S. J. Wallace, Phys. Rev. D 84, 074508 (2011).

[3] M. S. Mahbub, W. Kamleh, D. B. Leinweber, P. J. Moran, and A. G. Williams, Phys. Lett. B 707, 389 (2012).

[4] A. L. Kiratidis, W. Kamleh, D. B. Leinweber, and B. J. Owen, Phys. Rev. D 91, 094509 (2015).

[5] M. S. Mahbub, W. Kamleh, D. B. Leinweber, P. J. Moran, and A. G. Williams, Phys. Rev. D 87, 094506 (2013).

[6] B. J. Menadue, W. Kamleh, D. B. Leinweber, and M. S. Mahbub, Phys. Rev. Lett. 108, 112001 (2012).

[7] B. Blossier, M. Della Morte, G. von Hippel, T. Mendes, and R. Sommer, J. High Energy Phys. 04 (2009) 094.

[8] B. J. Owen, W. Kamleh, D. B. Leinweber, M. S. Mahbub, and B. J. Menadue, Phys. Rev. D 92, 034513 (2015).

[9] J. M. M. Hall, W. Kamleh, D. B. Leinweber, B. J. Menadue, B. J. Owen, A. W. Thomas, and R. D. Young, Phys. Rev. Lett. 114, 132002 (2015).

[10] B. J. Owen, W. Kamleh, D. B. Leinweber, M. S. Mahbub, and B. J. Menadue, Phys. Rev. D 91, 074503 (2015).

[11] B. J. Owen, J. Dragos, W. Kamleh, D. B. Leinweber, M. S. Mahbub, B. J. Menadue, and J. M. Zanotti, Phys. Lett. B 723, 217 (2013).

[12] J. Bulava, M. Donnellan, and R. Sommer, J. High Energy Phys. 01 (2012) 140.

[13] H.-W. Lin, S. D. Cohen, R. G. Edwards, and D. G. Richards, Phys. Rev. D 78, 114508 (2008).

[14] H.-W. Lin, S. D. Cohen, R. G. Edwards, K. Orginos, and D. G. Richards, arXiv:1005.0799.

[15] B. Yoon et al., Phys. Rev. D 93, 114506 (2016).
[16] S. Capitani, M. Della Morte, G. von Hippel, B. Jäger, A. Jüttner, B. Knippschild, H. B. Meyer, and H. Wittig, Phys. Rev. D 86, 074502 (2012).

[17] J. R. Green, J. W. Negele, A. V. Pochinsky, S. N. Syritsyn, M. Engelhardt, and S. Krieg, Phys. Rev. D 90, 074507 (2014).

[18] S. Capitani, M. Della Morte, D. Djukanovic, G. von Hippel, J. Hua, B. Jäger, B. Knippschild, H. B. Meyer, T. D. Rae, and H. Wittig, Phys. Rev. D 92, 054511 (2015).

[19] G. S. Bali, S. Collins, B. Gläßle, M. Göckeler, J. Najjar, R. H. Rödl, A. Schäfer, R. W. Schiel, W. Söldner, and A. Sternbeck, Phys. Rev. D 91, 054501 (2015).

[20] T. Bhattacharya, S. D. Cohen, R. Gupta, A. Joseph, H.-W. Lin, and B. Yoon, Phys. Rev. D 89, 094502 (2014).

[21] S. Dinter, C. Alexandrou, M. Constantinou, V. Drach, K. Jansen, and D. B. Renner, Phys. Lett. B 704, 89 (2011).

[22] V. G. Bornyakov et al., arXiv:1508.05916.

[23] W. Bietenholz et al., Phys. Lett. B 690, 436 (2010).

[24] W. Bietenholz et al., Phys. Rev. D 84, 054509 (2011).

[25] M. Constantinou, R. Horsley, H. Panagopoulos, H. Perlt, P. E. L. Rakow, G. Schierholz, A. Schiller, and J. M. Zanotti, Phys. Rev. D 91, 014502 (2015).

[26] K. U. Can, A. Kusno, E. V. Mastropas, and J. M. Zanotti, Lect. Notes Phys. 889, 69 (2015).

[27] S. Güsken, Nucl. Phys. B, Proc. Suppl. 17, 361 (1990).

[28] A. J. Chambers et al. (QCDSF/UKQCD, CSSM Collaborations), Phys. Rev. D 90, 014510 (2014).

[29] W. Detmold, W. Melnitchouk, J. W. Negele, D. B. Renner, and A. W. Thomas, Phys. Rev. Lett. 87, 172001 (2001).

[30] Y. Nakamura and H. Stüben, Proc. Sci., LATTICE2010 (2010) 040 [arXiv:1011.0199].

[31] P. A. Boyle, Comput. Phys. Commun. 180, 2739 (2009). 